\title{
A dynamic location problem with maximum decreasing capacities
}

\author{
Joana Dias • M. Eugénia Captivo · João Clímaco
}

Accepted: 24 January 2008 / Published online: 29 February 2008

(C) Springer-Verlag 2008

\begin{abstract}
In this paper a capacitated dynamic location problem with opening, closure and reopening of facilities is formulated and a primal-dual heuristic that can solve this problem is described. The problem formulated considers the situation where a facility is open (or reopens) with a certain maximum capacity that decreases as clients are assigned to that facility during its operating periods. This problem is $N P$-hard. Computational results are presented and discussed.
\end{abstract}

Keywords Dynamic location problems $\cdot$ Heuristics

\section{JEL Classification C61}

\section{Introduction}

Consider a network with a set $\mathcal{N}$ of nodes and a set $\mathcal{A}$ of $\operatorname{arcs}$. Set $\mathcal{N}$ can be divided in two disjoint subsets $\mathcal{N} 1$ and $\mathcal{N} 2$. Nodes in $\mathcal{N} 1$ are characterized by having a given

This research was partially supported by research project POCTI/ISFL-1/152 and POCTI/MAT/139/2001.

J. Dias $(\varangle) \cdot$ J. Clímaco

Faculdade de Economia and INESC-Coimbra, Universidade de Coimbra,

Av. Dias da Silva, 165, 3004-512 Coimbra, Portugal

e-mail: joana@fe.uc.pt

J. Clímaco

e-mail: jclimaco@inescc.pt

M. E. Captivo

Universidade de Lisboa, Faculdade de Ciências, Centro de Investigação Operacional,

Campo Grande, Bloco C6, Piso 4, 1749-016 Lisboa, Portugal

e-mail: mecaptivo@fc.ul.pt 
demand. Nodes in $\mathcal{N} 2$ can, potentially, be suppliers. They are characterized by having maximum limits on the demand they can serve. There is a fixed cost incurred by fixing the capacity of a supplier in a value greater than zero. There are also costs incurred by satisfying the demand of a node in $\mathcal{N} 1$ using the capacity of a node in $\mathcal{N} 2$. The objective is to decide which nodes in $\mathcal{N} 2$ will be used as suppliers and which arcs in $\mathcal{A}$ will be used to satisfy the demand of all nodes in $\mathcal{N} 1$, minimizing both fixed and assignment costs. As it is easily seen, this problem can be formulated as a capacitated location problem. Now imagine that the costs associated with arcs in $\mathcal{A}$ can change over time, or even that the set $\mathcal{A}$ itself can change over time (by insertion or deletion of arcs). It is also possible to imagine that the demand of nodes in $\mathcal{N} 1$ is time dependent. In this case, it is straightforward to conclude that the possibility of modifying the network design during the planning horizon should be considered. This means that there can exist nodes in $\mathcal{N} 2$ that are suppliers at time period $t$ but not at $t+1$ or $t-1$, or arcs that are used in one time period and not in the next one or at the previous one. This dynamic version of the problem can be formulated as a dynamic location problem that allows the reconfiguration of suppliers more than once during the planning horizon.

The problem studied in this paper has two important characteristics that distinguish it from the previous work done in this area: it is a capacitated dynamic location problem that considers the possibility of reconfiguring one location more than once during the planning horizon. This means that a facility can be open, closed and reopen more than once, which increases the flexibility of the model. Differentiation between the opening and the reopening of a facility is convenient because it allows the differentiation of the corresponding fixed costs (that can be clearly different). The model proposed also consider the existence of closing costs which, most of the times, cannot be ignored. Moreover it considers a different type of capacity restrictions: the existence of an initial maximum capacity that decreases as the facility serves clients. These kind of restrictions appear, for instance, when locating sanitary landfills that have a maximum capacity when are opened that diminishes as the solid waste is disposed.

There are several references in the literature that deal with capacitated location problems (see, for instance, Cornuejols et al. 1991; Sridharan 1995). It is more difficult to find references to the dynamic capacitated location problem than to the static version of the problem. Most of the references consider maximum capacity restrictions (see, for instance, Van Roy and Erlenkotter 1982; Saldanha da Gama 2002; Saldanha da Gama and Captivo 2002; Dias et al. 2006, 2007), different from the ones considered in this paper.

The primal-dual heuristic developed here is based on the work of Erlenkotter (1978), Van Roy and Erlenkotter (1982) and Guignard and Spielberg (1979). This heuristic is also an extension of the previous work done by the authors Dias et al. (2006) considering a different kind of capacity restrictions. It builds a pair of primal and dual solutions, trying to force the complementary conditions to be fulfilled.

In the next two sections the problem addressed is formulated, the corresponding linear dual problem is presented and the primal-dual heuristic is described. In Sect. 4 computational experiments are described and the results shown, in Sect. 5 some final comments are made and future work directions are pointed out. 


\section{Dynamic location problem with maximum decreasing capacity constraints}

Consider the following notation:

$J=\{1, \ldots, i, \ldots, n\}$ set of indices corresponding to the clients' locations;

$I=\{1, \ldots, j, \ldots, m\}$ set of indices corresponding to facilities' possible locations;

$T=$ number of time periods considered in the planning horizon $(1 \leq t \leq \xi \leq T)$;

$c_{i j}^{t}=$ cost of fully assigning client $j$ to facility $i$ in period $t$;

$F A_{i t}^{\xi}=$ fixed cost of opening a facility $i$ at the beginning of period $t$, and closing it at the end of period $\xi$ (the facility will be in operation from the beginning of $t$ to the end of $\xi$ );

$F R_{i t}^{\xi}=$ fixed cost of reopening a facility $i$ at the beginning of period $t$, and closing it at the end of period $\xi$ (the facility will be in operation from the beginning of $t$ to the end of $\xi$ );

$d_{j}^{t}=$ demand of client $j$ at period $t$;

$Q_{i}=$ maximum capacity of the facility located at $i$, at the time of (re) opening;

and let us define the variables:

$$
\begin{aligned}
& a_{i t}^{\xi}= \begin{cases}1 & \begin{array}{l}
\text { if facility } i \text { is opened at the beginning of period } t \\
\text { and stays open until the end of period } \xi \\
\text { otherwise }
\end{array}\end{cases} \\
& r_{i t}^{\xi}=\left\{\begin{array}{ll}
1 & \begin{array}{l}
\text { if facility } i \text { is reopened at the beginning } \\
\text { of period } t \text { and stays open until the end } \\
\text { of period } \xi \\
0
\end{array}
\end{array}, t>1\right.
\end{aligned}
$$

$x_{i j}^{t}=$ fraction of customer $j$ 's demand that is served by facility $i$ during period $t$.

Consider a situation where a service can be opened (or reopened) with a certain maximum capacity. As long as this facility serves clients' demand, its capacity decreases. Examples of facilities with this kind of behavior can be found, for instance, in sanitary landfills. When these facilities are opened, they can receive a maximum quantity of solid waste. This maximum capacity decreases during the life-period of the sanitary landfill, as it receives solid waste.

The dynamic location problem with maximum decreasing capacities that allows facilities to open, close and reopen more than once during the planning horizon will be formulated as DC-DLPOCR:

\section{DC-DLPOCR}

$$
\operatorname{Min} \sum_{t} \sum_{i} \sum_{j} c_{i j}^{t} x_{i j}^{t}+\sum_{t} \sum_{i} \sum_{\xi=t}^{T} F A_{i t}^{\xi} a_{i t}^{\xi}+\sum_{t} \sum_{i} \sum_{\xi=t}^{T} F R_{i t}^{\xi} r_{i t}^{\xi}
$$

subject to:

$$
\sum_{i} x_{i j}^{t}=1, \quad \forall j, t
$$




$$
\begin{aligned}
& \sum_{\tau=1}^{t} \sum_{\xi=t}^{T}\left(a_{i \tau}^{\xi}+r_{i \tau}^{\xi}\right)-x_{i j}^{t} \geq 0, \quad \forall i, j, t \\
& \sum_{\tau=1}^{t-1} \sum_{\xi=\tau}^{t-1} a_{i \tau}^{\xi}-\sum_{\xi=t}^{T} r_{i t}^{\xi} \geq 0, \quad \forall i, t \\
& \sum_{t=1}^{T} \sum_{\xi=t}^{T} a_{i t}^{\xi} \leq 1, \quad \forall i \\
& \sum_{\tau=1}^{t} \sum_{\xi=t}^{T}\left(a_{i \tau}^{\xi}+r_{i \tau}^{\xi}\right) \leq 1, \quad \forall i, t \\
& Q_{i} \sum_{\tau=1}^{t} \sum_{\xi=\tau}^{T}\left(a_{i \tau}^{\xi}+r_{i \tau}^{\xi}\right)-\sum_{\tau=1}^{t} \sum_{j} d_{j}^{\tau} x_{i j}^{\tau} \geq 0, \quad \forall i, t \\
& a_{i t}^{\xi} \in\{0,1\}, \quad \forall i, t, \xi \geq t \\
& r_{i t}^{\xi} \in\{0,1\}, \quad \forall i, t>1, \xi \geq t
\end{aligned}
$$

Constraints (2) guarantee that, in every time period, each client's demand is satisfied; constraints (3) assure that, in every time period, a client can only be assigned to facilities that are operational in that time period; constraints (4) and (6) impose that a facility can only be reopened at the beginning of period $t$ if it has already been open earlier and it is not in operation at the beginning of period $t$ and that, in every time period, only one facility can be open in each location; constraints (5) guarantee that a facility can only be opened once during the planning horizon. The model presented considers admissible the situation where a facility is closed even if its capacity has not been totally used. Restriction (7) considers that when a facility is reopened, its maximum capacity will be equal to $Q_{i}$ plus the remaining capacity the facility had when it was closed. It can be argued that this behavior is not admissible for some kinds of facilities. Thinking, for instance, of sanitary landfills it is easy to imagine that if a sanitary landfill is closed at period $t$ and reopened at period $t+1$, then its remaining capacity at the end of $t$ can be used. Nevertheless, if the sanitary landfill is reopened several time periods after its closure, its remaining capacity at the end of period $t$ will have been lost (because of all the closing and maintenance operations that need to be performed). The fixed opening and reopening costs of these kind of facilities are generally huge when compared with transportation and handling costs, so it is not expected that a facility with useful remaining capacity will be closed, unless the remaining capacity is insignificant when compared with $Q_{i}$. Furthermore, the decision maker is free to consider only the $a_{i \tau}^{\xi}$ and $r_{i \tau}^{\xi}$ variables he/she feels are needed. He/she can, for instance, consider variables such that $\xi-\tau$ is greater than a minimum time interval. For these reasons, the authors feel that the model presented has an acceptable behavior and can be considered useful in the resolution of many real problems, but are aware of the limitations of these capacity restrictions in some situations, especially because the fixed reopening costs do not reflect the time distance between the closure and the reopening periods. 


\subsection{Formulation of the dual problem}

Multiplying constraints (5) and (6) by -1 and associating dual variables $v_{j}^{t}$ with constraints (2), $w_{i j}^{t}$ with constraints (3), $u_{i}^{t}$ with constraints (4), $\rho_{i}$ with constraints (5), $\pi_{i}^{t}$ with constraints (6), $\lambda_{i}^{t}$ with constraints (7) and defining $w_{i j}^{t}=\max \left\{0, v_{j}^{t}-c_{i j}^{t}\right.$ $\left.-\sum_{\psi=t}^{T} d_{j}^{t} \lambda_{i}^{\psi}\right\}, \forall i, j, t$, the condensed dual of the linear relaxation of DC-DLPOCR can be formulated as:

\section{CDDC-DLPOCR}

$$
\operatorname{Max} \sum_{t} \sum_{j} v_{j}^{t}-\sum_{i} \rho_{i}-\sum_{t} \sum_{i} \pi_{i}^{t}
$$

subject to:

$$
\begin{gathered}
\sum_{j} \sum_{\tau=t}^{\xi} \max \left\{0, v_{j}^{\tau}-c_{i j}^{\tau}-\sum_{\psi=\tau}^{T} d_{j}^{\tau} \lambda_{i}^{\psi}\right\} \\
\leq F A_{i t}^{\xi}-\sum_{\tau=\xi+1}^{T} u_{i}^{\tau}+\rho_{i}+\sum_{\tau=t}^{\xi} \pi_{i}^{\tau}-Q_{i} \sum_{\tau=t}^{T} \lambda_{i}^{\tau}, \\
\forall i, t, \xi=t, \ldots, T \\
\sum_{j} \sum_{\tau=t}^{\xi} \max \left\{0, v_{j}^{\tau}-c_{i j}^{\tau}-\sum_{\psi=\tau}^{T} d_{j}^{\tau} \lambda_{i}^{\psi}\right\} \\
\leq F R_{i t}^{\xi}+u_{i}^{t}+\sum_{\tau=t}^{\xi} \pi_{i}^{\tau}-Q_{i} \sum_{\tau=t}^{T} \lambda_{i}^{\tau}, \\
\forall i, \quad t>1, \quad \xi=t, \ldots, T \\
u_{i}^{t}, \rho_{i}, \pi_{i}^{t}, \lambda_{i}^{t} \geq 0, \quad \forall i, t
\end{gathered}
$$

\subsection{Complementary conditions}

Let us define:

$$
\begin{aligned}
S A_{i t}^{\xi}= & F A_{i t}^{\xi}-\sum_{\tau=\xi+1}^{T} u_{i}^{\tau}+\rho_{i}+\sum_{\tau=t}^{\xi} \pi_{i}^{\tau}-\sum_{j} \sum_{\tau=t}^{\xi} \max \left\{0, v_{j}^{\tau}-c_{i j}^{\tau}-\sum_{\psi=\tau}^{T} d_{j}^{\tau} \lambda_{i}^{\psi}\right\} \\
& -Q_{i} \sum_{\tau=t}^{T} \lambda_{i}^{\tau}, \quad \forall i, t, \xi=t, \ldots, T
\end{aligned}
$$




$$
\begin{aligned}
S R_{i t}^{\xi}= & F R_{i t}^{\xi}+u_{i}^{t}+\sum_{\tau=t}^{\xi} \pi_{i}^{\tau}-\sum_{j} \sum_{\tau=t}^{\xi} \max \left\{0, v_{j}^{\tau}-c_{i j}^{\tau}-\sum_{\psi=\tau}^{T} d_{j}^{\tau} \lambda_{i}^{\psi}\right\} \\
& -Q_{i} \sum_{\tau=t}^{T} \lambda_{i}^{\tau}, \quad \forall i, t>1, \xi=t, \ldots, T \\
S_{i t}^{\xi}= & \min \left\{S A_{i t}^{\xi}, S R_{i t}^{\xi}\right\}, \quad \forall i, t, \quad \xi=t, \ldots, T
\end{aligned}
$$

The following complementary slackness conditions hold if in presence of optimal primal and dual feasible solutions (when there is no duality gap).

$$
\begin{aligned}
& \left(\sum_{\tau=1}^{t} \sum_{\xi=t}^{T}\left(a_{i \tau}^{\xi}+r_{i \tau}^{\xi}\right)-x_{i j}^{t}\right) w_{i j}^{t}=0, \quad \forall i, j, t \\
& \left(\sum_{\tau=1}^{t-1} \sum_{\xi=\tau}^{t-1} a_{i \tau}^{\xi}-\sum_{\xi=t}^{T} r_{i t}^{\xi}\right) u_{i}^{t}=0, \quad \forall i, t \\
& \left(\sum_{t=1}^{T} \sum_{\xi=t}^{T} a_{i t}^{\xi}-1\right) \rho_{i}=0, \quad \forall i \\
& \left(\sum_{\tau=1}^{t} \sum_{\xi=t}^{T}\left(a_{i \tau}^{\xi}+r_{i \tau}^{\xi}\right)-1\right) \pi_{i}^{t}=0, \quad \forall i, t \\
& S A_{i t}^{\xi} \cdot a_{i t}^{\xi}=0, \quad \forall i, t, \xi=t, \ldots, T \\
& S R_{i t}^{\xi} \cdot r_{i t}^{\xi}=0, \quad \forall i, t>1, \xi=t, \ldots, T \\
& \left(Q_{i} \sum_{\tau=1}^{t} \sum_{\xi=\tau}^{T}\left(a_{i \tau}^{\xi}+r_{i \tau}^{\xi}\right)-\sum_{\tau=1}^{t} \sum_{j} d_{j}^{\tau} x_{i j}^{\tau}\right) \lambda_{i}^{t}=0, \quad \forall i, t
\end{aligned}
$$

\section{Primal-dual heuristic}

The primal-dual heuristic developed to solve the problem formulated in the previous section builds admissible primal solutions based on admissible dual solutions, trying to force the complementary conditions to be satisfied. The heuristic functioning scheme is the following:

1. Initialisation of dual variables;

2. Dual ascent procedure for dual variables $v_{j}^{t}$;

3. Primal procedure;

4. Dual adjustment procedure for dual variables $\rho_{i}$. If the dual solution is changed go to 2;

5. Repeat the dual-primal adjustment procedure for variables $v_{j}^{t}$ until there is no improvement in the dual objective function value; 
6. Dual adjustment procedure for dual variables $\rho_{i}$. If the dual solution is changed go to 2;

7. Dual ascent procedure for dual variables $u_{i}^{t}$. If the dual solution is changed go to 2 ;

8. Dual descent procedure for dual variables $u_{i}^{t}$. If the dual solution is changed go to 2 ;

9. Dual ascent procedure for dual variables $\lambda_{i}^{t}$. If the dual solution is changed go to 2;

10. Dual descent procedure for dual variables $\lambda_{i}^{t}$. If the dual solution is changed go to 2;

11. Dual adjustment procedure for variables $\pi_{i}^{t}$. If the dual solution is changed go to 2 .

The heuristic will stop when the optimal solution is found (the pair of primal and dual solutions satisfies all complementary conditions), or when there are no improvements in either primal or dual objective function values. Dual variables are initialised as:

1. $v_{j}^{t}=\min _{i}\left\{c_{i j}^{t}\right\}, \quad \forall j, t ; \pi_{i}^{t}=0, \quad \forall i, t ;$

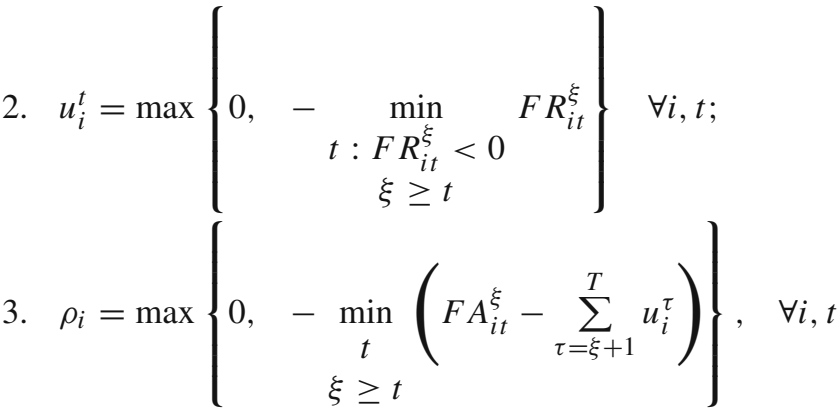

Step 2 of the primal-dual heuristic tries to increase all dual variables $v_{j}^{t},(j, t) \in$ $J^{+}, J^{+} \subset J \times T$. If this procedure is executed in step 2 of the heuristic, then $J^{+}$is the whole set $J \times T$. Whenever this procedure is executed within other procedure, the set $J^{+}$will be defined before the Dual Ascent procedure is called. This procedure is a straightforward adaptation of the one described in Van Roy and Erlenkotter, 1982. The only difference is in the updating step of slacks $S A_{i \tau}^{\xi}$ and $S R_{i \tau}^{\xi}$ : each time the value of $v_{j}^{t}$ is increased, slacks $S A_{i \tau}^{\xi}$ and $S R_{i \tau}^{\xi}, \tau \leq t \leq \xi$, have to be updated (its value will be decreased by the same amount the dual variable was increased, if $v_{j}^{t}$ is greater than or equal to $c_{i j}^{t}$ ). The assignment costs for period $t$ should be considered equal to $c_{i j}^{t}+d_{j}^{t} \sum_{\tau=t}^{T} \lambda_{i}^{\tau}$.

In most of the capacitated dynamic location problems, after deciding which facilities are open at each time period, the optimal value of the assignment variables can be calculated through the resolution of $T$ transportation problems. In the present problem, the resolution of $T$ transportation problems does not guarantee the calculation of the optimal assignments of clients to facilities, because the available capacity at period $t$ is dependant on the remaining available capacity at the end of period $t-1$. The 
resolution of the following linear programming problem guarantees the calculation of optimal assignments.

\section{PL1}

$$
\operatorname{Min} \sum_{t} \sum_{i} \sum_{j} c_{i j}^{t} x_{i j}^{t}
$$

subject to:

$$
\begin{gathered}
\sum_{i \in I_{t}^{+}} x_{i j}^{t}=1, \quad \forall j, t \\
Q_{i} \sum_{\tau=1}^{t} \sum_{\xi=\tau}^{T}\left(a_{i \tau}^{\xi}+r_{i \tau}^{\xi}\right)-\sum_{\tau=1}^{t} \sum_{j} d_{j}^{\tau} x_{i j}^{\tau} \geq 0, \quad \forall t, i \in I_{t}^{+} \\
x_{i j}^{t} \geq 0, \quad \forall j, t, i \in I_{t}^{+}
\end{gathered}
$$

\subsection{Dual adjustment procedure for variables $\rho_{i}$}

If it is possible for a variable $\rho_{i}$ to decrease its value, the dual objective function value will automatically increase. The value of variable $\rho_{i}$ can be decreased if $S A_{i \tau}^{\xi} \neq$ $0, \forall 1 \leq \tau \leq \xi$. Increasing the value of the dual variable $\rho_{i}$, increases all slacks $S A_{i \tau}^{\xi}$. The change in these slacks allows the increase of some $v_{j}^{t}$ that were blocked. However, variables $\rho_{i}$ have a coefficient of minus one in the dual objective function. Therefore, they should only be increased if the enhancement of variables $v_{j}^{t}$ is compensatory. It should be noted that it is worth trying to increase $\rho_{i}$ only if $S R_{i \tau}^{\xi} \neq 0$ and $S A_{i \tau}^{\xi}=0$. Otherwise, a change in the slack $S A_{i \tau}^{\xi}$ would not be reflected in dual variables $v_{j}^{t}$.

\section{Dual adjustment procedure for dual variables $\rho_{i}$}

1. $i \leftarrow 1$;

2. $\Delta \rho_{i} \leftarrow \min _{\tau \leq \xi}\left\{S A_{i \tau}^{\xi}\right\}$. If $\Delta \rho_{i}=0$ then continue. Else go to 7 .

3. $\Delta \rho_{i}=\max \left\{S R_{i \tau}^{\xi}: \exists(i, \tau, \xi) \in I_{R}^{+}\right.$with $S A_{i \tau}^{\xi}=0$ and $\left.S R_{i \tau}^{\xi} \neq 0\right\}$.

4. If $\Delta \rho_{i} \neq 0$ then $\rho_{i} \leftarrow \rho_{i}+\Delta \rho_{i} ; S A_{i \tau}^{\xi} \leftarrow S A_{i \tau}^{\xi}+\Delta \rho_{i}, \forall \tau, \xi \geq \tau$. Else go to 8 .

5. $J^{+}=\left\{(j, t): I_{j}^{t *}=\{i\}, \forall t\right\}$. Execute the dual ascent procedure for dual variables $v_{j}^{t}$.

$J^{+}=J \times T$. Execute the dual ascent procedure for dual variables $v_{j}^{t}$.

6. $\Delta \rho_{i}=\min _{\tau} S A_{i \tau}^{\xi}$.

$$
\xi \geq \tau
$$

7. $\Delta \rho_{i}=\min \left\{\Delta \rho_{i}, \rho_{i}\right\}$. If $\Delta \rho_{i} \neq 0$ then $S A_{i \tau}^{\xi} \leftarrow S A_{i \tau}^{\xi}-\Delta \rho_{i}, \quad \forall \tau, \xi \geq \tau ; \rho_{i} \leftarrow$ $\rho_{i}-\Delta \rho_{i}$.

8. If $i=m$ then stop. Else $i \leftarrow i+1$; go to 2 .

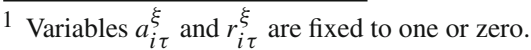


3.2 Dual-primal adjustment procedure for variables $v_{j}^{t}$

The dual-primal adjustment procedure for variables $v_{j}^{t}$ detects violations of the complementary conditions (14), and decreases the values of some variables $v_{j}^{t}$, allowing other variables $v_{j}^{t}$ to increase. This procedure can reduce the number of violations of complementary conditions (14) and, at the same time, can improve the value of the dual objective function.

Consider the following sets:

$$
\begin{aligned}
I_{j}^{t *} & =\left\{i: \exists(\tau, \xi) \quad \text { with } \tau \leq t \leq \xi \mid(i, \tau, \xi) \in I^{*} \text { and } v_{j}^{t} \geq c_{i j}^{t}\right\} \\
I_{j}^{t+} & =\left\{i: i \in I_{t}^{+} \text {and } v_{j}^{t}>c_{i j}^{t}\right\} \\
J_{i}^{t+} & =\left\{(j, \tau): I_{j}^{\tau *}=\{i\} \quad \text { and } \quad(i, \gamma, \xi) \notin I^{*}, \gamma \leq \xi \leq \tau<t \quad \text { or } t<\tau \leq \gamma \leq \xi\right\}
\end{aligned}
$$

The set $I_{j}^{t+}$ indicates, for each client $j$, all operating facilities during period $t$ such that $v_{j}^{t}$ is greater than the assignment $\operatorname{cost} c_{i j}^{t}$. A violation of the complementary condition (14) is detected by the existence of, at least, one pair $(j, t)$ such that the number of elements in $I_{j}^{t+}$ is greater than one. Decreasing the value of a variable $v_{j}^{t}$ such that the number of elements in $I_{j}^{t+}$ is greater than one, means that at least slacks $S_{i \tau}^{\xi}, \tau \leq t \leq \xi$, will be increased for two distinct facilities. This may promote the increase in the dual objective function. The set $J_{i}^{t+}$ represents all variables $v_{j}^{t}$ whose value can be increased with the rise of slack $S_{i \tau}^{\xi}, \tau \leq t \leq \xi$. This procedure is a straightforward adaptation of the one described in Erlenkotter 1978 and Van Roy and Erlenkotter (1982) taking into account the remarks of Saldanha da Gama and Captivo (2002).

\subsection{Dual ascent procedure for variables $u_{i}^{t}$}

Increasing variables $u_{i}^{t}$, increases slacks $S R_{i t}^{\xi}, \xi \geq t$, but at the same time diminishes slacks $S A_{i \tau}^{\xi}, \tau \leq \xi<t$. If the procedure is able to increase slacks $S_{i \tau}^{\xi}$ that are blocking variables $v_{j}^{t}$, decreasing $S_{i \tau}^{\xi}$ that are not blocking any variable $v_{j}^{t}$, then it will be possible to improve the dual objective function value. If there is $S R_{i t}^{\xi}=0$ and $S A_{i t}^{\xi} \neq 0$, then the increase in $u_{i}^{t}$ can be of help. This situation occurs, for instance, when $(i, t, \xi) \in I_{A}^{+}$with $S A_{i t}^{\xi} \neq 0$ and $S R_{i t}^{\xi}=0$. In this case, $S R_{i t}^{\xi}$ should not increase more than $S A_{i t}^{\xi}-S R_{i t}^{\xi}$, because any further increase will not change the value of $S_{i t}^{\xi}$. On the other hand, variable $u_{i}^{t}$ cannot grow more than the minimum value of $S A_{i \tau}^{\xi}, \forall \tau \leq \xi<t$, so that the dual solution remains admissible. Increasing variable $u_{i}^{t}$ can diminish the number of violations of complementary conditions (18). Consider variables $u_{i}^{t}$ organized as a sequence of pairs $(i, t)$. 


\section{Dual ascent procedure for dual variables $u_{i}^{t}$}

1. Initialise $(i, t) \leftarrow(i, t)_{1} ; q \leftarrow 1$.

2. $\xi \leftarrow t ; \Delta u_{i}^{t} \leftarrow 0 ; \delta \leftarrow 0$.

3. If $S R_{i t}^{\xi}=0$ and $S A_{i t}^{\xi} \neq 0$, then $\Delta u_{i}^{t} \leftarrow \max \left\{\Delta u_{i}^{t}, S A_{i t}^{\xi}\right\}$ and $\delta \leftarrow 1$.

4. If $\xi=T$ go to 5 , else $\xi \leftarrow \xi+1$, go to 3 .

5. If $\delta=0$, go to 7. Else $\Delta u_{i}^{t} \leftarrow \min \left\{\Delta u_{i}^{t}, \min _{\tau \leq \gamma<t} S A_{i \tau}^{\gamma}\right\}$,

$S R_{i t}^{\xi} \leftarrow S R_{i t}^{\xi}+\Delta u_{i}^{t}, \forall \xi \geq t$.

$S A_{i \tau}^{\xi} \leftarrow S A_{i \tau}^{\xi}-\Delta u_{i}^{t}, \forall \tau \leq \xi<t$ and $u_{i}^{t} \leftarrow u_{i}^{t}+\Delta u_{i}^{t}$.

6. $J^{+}=\left\{(j, t): I_{j}^{t *}=\{i\}, \forall t\right\}$. Execute the dual ascent procedure for variables $v_{j}^{t}$.

$J^{+}=J \times T$. Execute the dual ascent procedure for variables $v_{j}^{t}$.

7. If $q=m \times T$ then stop. Else $q \leftarrow q+1$; $(i, t) \leftarrow(i, t)_{q}$, go to 2 .

\subsection{Dual descent procedure for variables $u_{i}^{t}$}

Decreasing $u_{i}^{t}$ will decrease slacks $S R_{i t}^{\xi}, \xi \geq t$, and increase slacks $S A_{i \tau}^{\xi}, \tau \leq \xi<t$. To guarantee the admissibility of the dual solution, variable $u_{i}^{t}$ can only be decreased if $S R_{i t}^{\xi}>0, \forall \xi \geq t$. If the procedure is able to increase slacks $S_{i \tau}^{\xi}$ that are blocking dual variables $v_{j}^{t}$ and decrease slacks that do not influence $v_{j}^{t}$ values, then it is possible to improve the dual objective function value.

\section{Dual descent procedure for variables $u_{i}^{t}$}

1. Initialise $(i, t) \leftarrow(i, t)_{1} ; q \leftarrow 1$.

2. If $u_{i}^{t}=0$ go to 6 ; Otherwise, $\Delta u_{i}^{t} \leftarrow 0$; $\delta \leftarrow 0$.

3. If $S R_{i t}^{\xi}>0, \quad \forall \xi \geq t$, then $\Delta u_{i}^{t} \leftarrow \min _{\xi \geq t}\left\{S R_{i t}^{\xi}\right\}$ and $\delta \leftarrow 1$.

4. If $\delta=0$ go to 6. Else $\Delta u_{i}^{t} \leftarrow \min \left\{\Delta u_{i}^{t}, u_{i}^{t}\right\}$;

$S R_{i t}^{\xi} \leftarrow S R_{i t}^{\xi}-\Delta u_{i}^{t}, \forall \xi \geq t$.

$S A_{i \tau}^{\xi} \leftarrow S A_{i \tau}^{\xi}+\Delta u_{i}^{t}, \forall \tau \leq \xi<t$ and $u_{i}^{t} \leftarrow u_{i}^{t}-\Delta u_{i}^{t}$.

5. $J^{+}=\left\{(j, t): I_{j}^{t *}=\{i\}, \forall t\right\}$. Execute the dual ascent procedure for variables $v_{j}^{t}$. $J^{+}=J \times T$. Execute the dual ascent procedure for variables $v_{j}^{t}$.

6. If $q=m \times T$ then stop. Else $q \leftarrow q+1$; $(i, t) \leftarrow(i, t)_{q}$, go to 2 .

3.5 Dual ascent procedure for variables $\lambda_{i}^{t}$

Variable $\lambda_{i}^{t}$ influences the value of all slacks $S A_{i \tau}^{\xi}$ and $S R_{i \tau}^{\xi}, \tau \leq t$. Consider the following definitions:

$$
\begin{aligned}
& \Delta= \max _{j \in J}\left\{\frac{1}{d_{j}^{\tau}} \max \left\{0, v_{j}^{\tau}-c_{i j}^{\tau}-\sum_{\xi=\tau}^{T} d_{j}^{\tau} \lambda_{i}^{\xi}\right\}\right\} ; \\
& J 1(t)=\left\{j \in J: v_{j}^{t}-c_{i j}^{t}-\sum_{\xi=t}^{T} d_{j}^{t} \lambda_{i}^{\xi} \leq \delta d_{j}^{t}\right\} .
\end{aligned}
$$


Proposition 1 If variable $\lambda_{i}^{t}$ is increased by $\left.\left.\delta \in\right] 0, \Delta\right]$, then slacks $S A_{i \tau}^{\xi}$ and $S R_{i \tau}^{\xi}$, $\tau \leq t$, will be changed by:

$$
\begin{aligned}
& \Phi(\delta, \tau, \xi) \\
& =\sum_{s=\tau}^{\min \{\xi, t\}}\left(\sum_{j \in J 1(s)} \max \left\{0, v_{j}^{s}-c_{i j}^{s}-\sum_{\psi=s}^{T} d_{j}^{s} \lambda_{i}^{\psi}\right\}+\sum_{j \notin J 1(s)} \delta d_{j}^{s}\right)-\delta Q_{i}
\end{aligned}
$$

Proof If $\lambda_{i}^{t}$ is increased by $\left.\left.\delta \in\right] 0, \Delta\right]$, all sums $\sum_{\psi=s}^{T} d_{j}^{s} \lambda_{i}^{\psi}$, with $s \leq t$, will be increased by $d_{j}^{s} \delta$. These sums influence the values of all slacks $S A_{i \tau}^{\xi}$ and $S R_{i \tau}^{\xi}$, with $\tau \leq t$. If $t>\xi$ then all sums with $\tau \leq s \leq \xi$ have to be taken into account. If $t \leq \xi$, then only sums with $\tau \leq s \leq t$ will change (sums with $s>t$ will not be altered). For each $s, \tau \leq s \leq \min \{\xi, t\}, v_{j}^{s}-c_{i j}^{s}-\sum_{\psi=s}^{T} d_{j}^{s} \lambda_{i}^{\psi}$ with $j \in J 1(s)$ will become less than or equal to zero (and the corresponding $w_{i j}^{s}$ variable will be equal to zero). For all $j \notin J 1(s)$, variables $w_{i j}^{s}$ will be decreased by $d_{j}^{s} \delta$. Dual variable $\lambda_{i}^{t}$ influences all slacks $S A_{i \tau}^{\xi}$ and $S R_{i \tau}^{\xi}, \tau \leq t$, not only due to sums $\sum_{\psi=s}^{T} d_{j}^{s} \lambda_{i}^{\psi}$, with $\tau \leq s \leq t$, but also due to the sum $Q_{i} \sum_{\psi=\tau}^{T} \lambda_{i}^{\psi}$. This sum will be increased by $\delta Q_{i}$. Therefore, it can be concluded that the total change in slacks $S A_{i \tau}^{\xi}$ and $S R_{i \tau}^{\xi}, \tau \leq t$, due to a change $\delta$ in dual variable $\lambda_{i}^{t}$ is given by $\Phi(\delta, \tau, \xi)$.

As can be seen by expression (25), slacks influenced by the increase in the dual variable will have different behaviors: some can be increased while others can be decreased.

Proposition 2 Consider that $\lambda_{i}^{t}$ is increased by $\delta^{\prime}$, with $\delta^{\prime}>\Delta$, being the resulting slacks $S A \prime_{i \tau}^{\xi}$ and $S R_{i \tau}^{\xi}$, with $\tau \leq t$. It is possible to find $\left.\left.\delta \in\right] 0, \Delta\right]$ such that if $\lambda_{i}^{t}$ is increased by $\delta$ instead of $\delta^{\prime}$, the resulting values of all slacks $S A_{i \tau}^{\xi}$ and $S R_{i \tau}^{\xi}$, with $\tau \leq t$, will be greater than or equal to $S A_{i \tau}^{\xi}$ and $S R_{i \tau}^{\xi}$, with $\tau \leq t$.

The proof follows directly from proposition 1 and the definition of $\Delta$. Proposition 1 motivates the following dual ascent procedure for variables $\lambda_{i}^{t}$.

\section{Dual ascent procedures for variables $\lambda_{i}^{t}$}

1. $t \leftarrow 1$;

2. $i \leftarrow 1 ; \delta^{\prime} \leftarrow+\infty$;

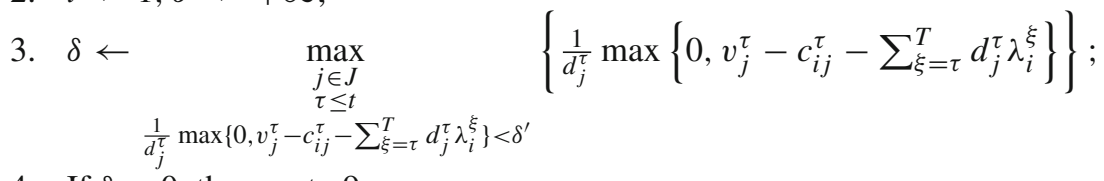

4. If $\delta=0$, then go to 9 . 
5. Compute $J 1(t)$ as in (24). If $\exists S A_{i \tau}^{\xi}$ or $S R_{i \tau}^{\xi}, \tau \leq t$, such that $S A_{i \tau}^{\xi}+\Phi(\delta, \tau, \xi)<$ 0 or $S R_{i \tau}^{\xi}+\Phi(\delta, \tau, \xi)<0$ go to 6 . Else go to 7 .

6. If $\delta^{\prime}=0$, then go to 9 . Else $\delta^{\prime} \leftarrow \delta$. Go to 4 .

7. $\lambda_{i}^{t} \leftarrow \lambda_{i}^{t}+\delta ; S A_{i \tau}^{\xi} \leftarrow S A_{i \tau}^{\xi}+\Phi(\delta, \tau, \xi)$ and $S R_{i \tau}^{\xi} \leftarrow S R_{i \tau}^{\xi}+\Phi(\delta, \tau, \xi), \forall \tau \leq t$.

8. Execute the dual ascent procedure for variables $v_{j}^{t}$.

9. $i \leftarrow i+1$; if $i>m$ then go to 10 . Else go to 3 .

10. $t \leftarrow t+1$; if $t>T$ then stop. Else go to 2 .

\subsection{Dual descent procedure for variables $\lambda_{i}^{t}$}

A decrease in the dual variable $\lambda_{i}^{t}$ will increase all values $v_{j}^{\tau}-c_{i j}^{\tau}-\sum_{\xi=\tau}^{T} d_{j}^{\tau} \lambda_{i}^{\xi}, \forall \tau \leq t$.

Proposition 3 If $\lambda_{i}^{t}$ is decreased by $\delta$, with:

$$
0<\delta \leq \min _{\substack{\tau \leq t \\ j \in J}}\left\{-\frac{v_{j}^{\tau}-c_{i j}^{\tau}-\sum_{\xi=\tau}^{T} d_{j}^{\tau} \lambda_{i}^{\xi}}{d_{j}^{\tau}-c_{i j}^{\tau}-\sum_{\xi=\tau}^{T} d_{j}^{\tau} \lambda_{i}^{\xi}<0}\right\},
$$

then all slacks $S A_{i \tau}^{\xi}$ and $S R_{i \tau}^{\xi}$, with $\tau \leq t$, will be changed by:

$$
\Omega(\delta, \tau, \xi)=\delta\left(Q_{i}-\sum_{\psi=\tau}^{\min \{\xi, t\}} \underset{j \in J}{v_{j}^{\psi}-c_{i j}^{\psi}-\sum_{\varsigma=\psi}^{T} d_{j}^{\psi} \lambda_{i}^{\varsigma} \geq 0} d_{j}^{\psi}\right) .
$$

Proof If $\lambda_{i}^{t}$ is decreased by $\delta$, all values $v_{j}^{s}-c_{i j}^{s}-\sum_{\psi=s}^{T} d_{j}^{s} \lambda_{i}^{\psi}, s \leq t$, will be increased by $\delta d_{j}^{s}$. To guarantee that $v_{j}^{s}-c_{i j}^{s}-\sum_{\psi=s}^{T} d_{j}^{s} \lambda_{i}^{\psi}<0$ will remain less than or equal to zero:

$$
v_{j}^{s}-c_{i j}^{s}-\sum_{\psi=s}^{T} d_{j}^{s} \lambda_{i}^{\psi}+\delta d_{j}^{s} \leq 0 \Leftrightarrow \delta \leq-\frac{v_{j}^{s}-c_{i j}^{s}-\sum_{\psi=s}^{T} d_{j}^{s} \lambda_{i}^{\psi}}{d_{j}^{s}} .
$$

Therefore, the upper limit defined by (26) guarantees that for all $j$ and $s$ such that $v_{j}^{s}-c_{i j}^{s}-\sum_{\psi=s}^{T} d_{j}^{s} \lambda_{i}^{\psi}<0$, this value will continue smaller than zero. For each slack $S A_{i \tau}^{\xi}$ and $S R_{i \tau}^{\xi}$, with $\tau \leq t$, all values $v_{j}^{s}-c_{i j}^{s}-\sum_{\psi=s}^{T} d_{j}^{s} \lambda_{i}^{\psi} \geq 0$, with $s \leq \min \{\xi, t\}$, will be increased by $\delta d_{j}^{S}$. Each of these slacks is also influenced by the decrease $\delta Q_{i}$ in sum $Q_{i} \sum_{\psi=\tau}^{T} \lambda_{i}^{\psi}$. Therefore $\Omega(\delta, \tau, \xi)$ expresses the change occurred in slacks $S A_{i \tau}^{\xi}$ and $S R_{i \tau}^{\xi}$, with $\tau \leq t$, due to a decrease $\delta$ in dual variable $\lambda_{i}^{t}$.

Proposition 3 motivates the following dual descent procedure for variables $\lambda_{i}^{t}$. 


\section{Dual descent procedure for variables $\lambda_{i}^{t}$}

1. $t \leftarrow 1$;

2. $i \leftarrow 1$;

3. $\delta \leftarrow \min \left\{\lambda_{i}^{t}, \min _{\substack{\tau \leq t \\ j \in J}}^{\substack{\tau \\ v_{j}^{\tau}-c_{i j}^{\tau}-\sum_{\xi=\tau}^{T} d_{j}^{\tau} \lambda_{i}^{\xi}<0}}\left\{-\frac{v_{j}^{\tau}-c_{i j}^{\tau}-\sum_{\xi=\tau}^{T} d_{j}^{\tau} \lambda_{i}^{\xi}}{d_{j}^{\tau}}\right\}\right\}$ If $\delta=0$, then go to 7.

4. If $S A_{i \tau}^{\xi}+\Omega(\delta, \tau, \xi)<0$ or $S R_{i \tau}^{\xi}+\Omega(\delta, \tau, \xi)<0$, for some $\tau \leq t$, then: $\delta \leftarrow \min _{\substack{\tau \leq t \\ \Omega(\delta, \tau, \xi)<0}}\left\{-\frac{S A_{i \tau}^{\xi}}{\Omega(\delta, \tau, \xi) / \delta},-\frac{S R_{i \tau}^{\xi}}{\Omega(\delta, \tau, \xi) / \delta}\right\}$.

5. If $\delta=0$ go to 7. Else $S A_{i \tau}^{\xi} \leftarrow S A_{i \tau}^{\xi}+\Omega(\delta, \tau, \xi)$ and $S R_{i \tau}^{\xi} \leftarrow S R_{i \tau}^{\xi}+$ $\Omega(\delta, \tau, \xi), \forall \tau \leq t . \lambda_{i}^{t} \leftarrow \lambda_{i}^{t}+\delta$.

6. Execute the dual ascent procedure for variables $v_{j}^{t}$.

7. $i \leftarrow i+1$; if $i>m$ then go to 8. Else go to 3 .

8. $t \leftarrow t+1$; if $t>T$ then stop. Else go to 2 .

3.7 Dual adjustment procedure for variables $\pi_{i}^{t}$

Increasing the value of $\pi_{i}^{t}$ will increase slacks $S_{i \tau}^{\xi}, \tau \leq t \leq \xi$. If there are slacks $S_{i \tau}^{\xi}, \tau \leq t \leq \xi$ that are blocking dual variables $v_{j}^{t}$, then it is possible to improve the value of the dual objective function. However it is only worth to increase $\pi_{i}^{t}$ if the change in dual variables $v_{j}^{t}$ compensates the loss of $\pi_{i}^{t}$ in the objective function value (the variable $\pi_{i}^{t}$ has a coefficient of minus one). If the procedure is able to diminish the value of $\pi_{i}^{t}$, maintaining the dual solution feasibility, then there is an immediate improvement in the dual objective function value.

Consider variables $\pi_{i}^{t}$ organized as a sequence of pairs $(i, t)$, and $M$ a large positive number.

\section{Dual adjustment procedure for variables $\pi_{i}^{t}$}

1. Initialise $(i, t) \leftarrow(i, t)_{1} ; q \leftarrow 1$.

2. $\Delta \pi_{i}^{t}=\min _{\tau \leq t \leq \xi} S_{i \tau}^{\xi}$. If $\Delta \pi_{i}^{t} \neq 0$, then go to 6. Else $\Delta \pi_{i}^{t} \leftarrow M$.

3. $S_{i \tau}^{\xi} \leftarrow S_{i \tau}^{\xi}+\Delta \pi_{i}^{t}, \forall \tau \leq t \leq \xi ; \pi_{i}^{t}=\pi_{i}^{t}+\Delta \pi_{i}^{t}$.

4. $J^{+}=\left\{(j, t): I_{j}^{t *}=\{i\}, \forall t\right\}$. Execute the dual ascent procedure for variables $v_{j}^{t}$. $J^{+}=J \times T$. Execute the dual ascent procedure for variables $v_{j}^{t}$.

5. $\Delta \pi_{i}^{t}=\min _{\tau \leq t \leq \xi} S_{i \tau}^{\xi}$. 
6. $\Delta \pi_{i}^{t}=\min \left\{\Delta \pi_{i}^{t}, \pi_{i}^{t}\right\}$. If $\Delta \pi_{i}^{t} \neq 0$ then $S_{i \tau}^{\xi} \leftarrow S_{i \tau}^{\xi}-\Delta \pi_{i}^{t}, \forall \tau \leq t \leq \xi$ and $\pi_{i}^{t}=\pi_{i}^{t}-\Delta \pi_{i}^{t}$

7. If $q=m \times T$ then stop. Else $q \leftarrow q+1$; $(i, t) \leftarrow(i, t)_{q}$, go to 2 .

\subsection{Primal procedure}

The primal procedure, here developed, guarantees the calculation of a primal admissible solution, if one exists for DC-PLDOCR. It begins by calculating a solution for the uncapacitated problem. When it is necessary to open more services during a period $t$, the procedure calculates the cost of opening facilities not in operation during that time period, but also the cost of opening or reopening facilities before period $t$. Every time a facility is (re) open, its capacity is increased. Consider the following notation:

$$
\begin{aligned}
& I^{*}=\left\{(i, \tau, \xi): S_{i \tau}^{\xi}=0\right\} \\
& I_{t}^{*}=\left\{i:(i, \tau, \xi) \in I^{*} \text { and } \tau \leq t \leq \xi\right\} \\
& I_{t}^{+}=\{i: \text { facility } i \text { is open during period } t\} \\
& I_{A}^{+}=\left\{(i, \tau, \xi): a_{i \tau}^{\xi}=1\right\} \\
& I_{R}^{+}=\left\{(i, \tau, \xi): r_{i \tau}^{\xi}=1\right\} \\
& h_{i}^{t}=\text { smallest cost incurred by opening a facility } i \notin I_{t}^{+} \text {during period } t . \\
& p_{i}^{t}=\text { smallest cost incurred by reopening a facility } i \in I_{t}^{+} \text {at the beginning of a } \\
& \text { period } t^{\prime}<t \text {. } \\
& \text { Cap }{ }_{i}^{t}=\text { Maximum capacity of facility } i \text { at the beginning of time period } t .
\end{aligned}
$$

\section{DC-DLPOCR primal procedure}

1. $I_{A}^{+}=I_{R}^{+}=\emptyset \cdot I_{t}^{+}=\emptyset, \forall t$. Build sets $I^{*}$ and $I_{t}^{*}$. Num $=0$;

2. For $t=1, \ldots, T$, include in set $I_{t}^{+}$all facilities $i$ such that $\exists j: v_{j}^{t} \geq c_{i j}^{t}$ and $v_{j}^{t}<$ $c_{i^{\prime} j}^{t}, \forall i^{\prime} \neq i$

3. For each client $j$ such that $v_{j}^{t}<c_{i j}^{t}, \forall i \in I_{t}^{+}$, include in set $I_{t}^{+}$facility $i$ such that $c_{i j}^{t}=\min _{v_{j}^{t} \geq c_{i^{\prime} j}^{t}} c_{i^{\prime} j}^{t}$. Num $=\mathrm{Num}+1$. If Num $=1$ then $I_{A}^{+}=I_{R}^{+}=\emptyset . I_{t}^{*}=I_{t}^{+}$ and $I_{t}^{+}=\emptyset, \forall t$, go to 2 . Else continue.

4. Build sets $I_{A}^{+}$and $I_{R}^{+}$. Update $I_{t}^{+}$. For $t=1, \ldots, T$, assign each client $j$ to facility $i^{\prime} \in I_{t}^{+}$such that $c_{i^{\prime} j}^{t}=\min _{i \in I}\left\{c_{i j}^{t}\right\}$.

5. Test complementary slackness conditions.

6. Solve problem PL1 optimally using a general solver. If PL1 has no admissible solutions, go to 7 . Else stop.

7. $t \leftarrow$ 1. $\operatorname{Cap}_{i}^{1} \leftarrow Q_{i}, \forall i \in I_{1}^{+}$and $\mathrm{Cap}_{i}^{1} \leftarrow 0, \forall i \notin I_{1}^{+}$.

8. $D \leftarrow \sum_{j} d_{j}^{t} ; \quad C \leftarrow \sum_{i \in I_{t}^{+}} \operatorname{Cap}_{i}^{t}$. If $D \leq C$ then go to 13 .

9. Calculate $h_{i}^{t}, \forall i \notin I_{t}^{+}$and $p_{i}^{t}, \forall i \in I_{t}^{+} . h_{i}^{t} \leftarrow+\propto, \forall i \in I_{t}^{+}$and $p_{i}^{t} \leftarrow+\propto$, $\forall i \notin I_{t}^{+}$.

10. Choose $i$ ' such that $\min \left\{h_{i^{\prime}}^{t}, p_{i^{\prime}}^{t}\right\}=\min \left\{h_{t}^{\prime}, p_{t}^{\prime}\right\}$ where $h_{t}^{\prime}=\min _{i \in I}\left\{h_{i}^{t}\right\}$ and $p_{t}^{\prime}=\min _{i \in I}\left\{p_{i}^{t}\right\}$.

11. Rebuild sets $I_{A}^{+}, I_{R}^{+}, I_{t}^{+}, \forall t$ and recalculate $\mathrm{Cap}_{i}^{t}, \forall i$, and $C$ according to the choice made in 10 . 


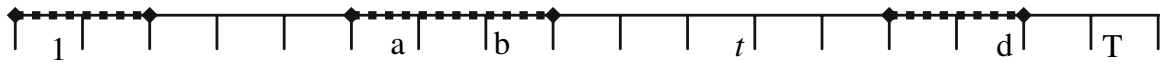

Fig. 1 represents facility $i$ functioning time periods

12. If $D>C$ then go to 9 . Else continue.

13. Solve one transportation problem considering as sources the set $J$ of clients (with supplies $d_{j}^{t}$ ), as destinations the set $I_{t}^{+}$(with demands $\operatorname{Cap}_{i}^{t}$ ), and unit transportation costs given by $c_{i j}^{t} / d_{j}^{t}$. Consider the values of the transportation variables designated by $x_{i j}^{\prime t}$.

14. $\operatorname{Cap}_{i}^{t} \leftarrow \operatorname{Cap}_{i}^{t}-\sum_{j \in J} x_{i j}^{\prime t}, \forall i \in I_{t}^{+}$.

15. $t \leftarrow t+1$; if $t>T$, then go to 6. Else continue.

16. If $\exists(i, t, \xi) \in I_{A}^{+} \cup I_{R}^{+}$, then $\operatorname{Cap}_{i}^{t} \leftarrow \operatorname{Cap}_{i}^{t-1}+Q_{i}$. Else $\operatorname{Cap}_{i}^{t} \leftarrow \operatorname{Cap}_{i}^{t-1}$. Go to 8 .

In the primal procedure, steps 4 and 5 require special attention. As a matter of fact, building sets $I_{A}^{+}$and $I_{R}^{+}$is much more complicated than building set $I^{+}$as described in Dynaloc Van Roy and Erlenkotter (1982) taking into account the remarks of Saldanha da Gama and Captivo (2002). If, for a facility $i \in I_{t}^{+}, S_{i \tau}^{\xi}=0, \tau \leq t \leq \xi$ for more than one pair $(\tau, \xi)$, the choice of which variable to include in set $I_{A}^{+}$or $I_{R}^{+}$is not trivial. For each facility $i$, these procedures include in $I_{A}^{+}$or $I_{R}^{+}$variables guaranteeing that facility $i$ will be open at least during periods $t$ such that $i \in I_{t}^{+}$, and that satisfy constraints (4)-(6).

Considering time periods $a, b, c, d$ (Fig. 1) defined formally as ${ }^{2}$

$$
\begin{aligned}
& b=\max \left\{0, \max _{t^{\prime}<t}\left\{t^{\prime}: i \in I_{t^{\prime}}^{+}\right\}\right\} ; a=t^{\prime} \text { such that }\left(i, t^{\prime}, b\right) \in I_{A}^{+} \cup I_{R}^{+} ; \\
& c=\min \left\{T+1, \min _{t^{\prime}>t}\left\{t^{\prime}: i \in I_{t^{\prime}}^{+}\right\}\right\} ; \quad d=t^{\prime} \text { such that }\left(i, c, t^{\prime}\right) \in I_{A}^{+} \cup I_{R}^{+} ;
\end{aligned}
$$

the calculation of $h_{i}^{t}$ is made as follows:

\section{Calculation of $h_{i}^{t}$ for $i \notin I_{t}^{+}$}

1. If $b=0$ and $c=T+1$ then $F_{i}^{t} \leftarrow \min \left\{F A_{i \tau}^{\xi}: 1 \leq \tau \leq t \leq \xi \leq T\right\}$. Stop.

2. If $b=0$ and $c \leq T$ then

$$
\begin{aligned}
& F_{i}^{t} \leftarrow \min \left\{\min \left\{F A_{i \tau}^{\xi}-F A_{i c}^{d}+F R_{i c}^{d}: 1 \leq \tau \leq t \leq \xi<c\right\}\right. \text {, . Stop. } \\
& \left.\min \left\{F A_{i \tau}^{d}-F A_{i c}^{d}: 1 \leq \tau \leq t\right\}\right\}
\end{aligned}
$$

3. If $b>0$ and $c=T+1$ then go to 4 . Else go to 7 .

4. If $(i, a, b) \in I_{A}^{+}$then go to 5. Else go to 6 .

5. $F_{i}^{t} \leftarrow \min _{\xi}\left\{\min \left\{F A_{i a}^{\xi}-F A_{i a}^{b}: t \leq \xi \leq T\right\}\right.$. Stop.

$$
\left.\min \left\{F R_{i \tau}^{\xi}: b<\tau \leq t \leq \xi \leq T\right\}\right\}
$$

\footnotetext{
2 Time period $\mathrm{b}$ represents the time period before and nearest to $t$ such that facility $\mathrm{i}$ is operating. Time period c represents the time period after and nearest to $t$ such that facility $\mathrm{i}$ is operating.
} 
6. $F_{i}^{t} \leftarrow \min _{i \min }\left\{F R_{i a}^{\xi}-F R_{i a}^{b}: t \leq \xi \leq T\right\}$, . Stop.
$\left.\min \left\{F R_{i \tau}^{\xi}: b<\tau \leq t \leq \xi \leq T\right\}\right\}$

7. If $(i, a, b) \in I_{A}^{+}$then go to 8. Else go to 9 .

8. $F_{i}^{t} \leftarrow \min _{\xi}\left\{F A_{i a}^{d}-F A_{i a}^{b}-F R_{i c}^{d}, \min \left\{F R_{i \tau}^{\xi}, b<\tau \leq t \leq \xi<c\right\}\right.$, . Stop.

9.

$$
\begin{aligned}
& F_{i}^{t} \leftarrow \min _{\xi}\left\{F R_{i a}^{d}-F R_{i a}^{b}-F R_{i c}^{d}, \min \left\{F R_{i \tau}^{\xi}, b<\tau \leq t \leq \xi<c\right\}\right. \text {, ; Stop. } \\
& \left.\min \left\{F R_{i a}^{\xi}-F R_{i a}^{b}, t \leq \xi<c\right\}, \min \left\{F R_{i \tau}^{d}-F R_{i c}^{d}, b<\tau \leq t\right\}\right\}
\end{aligned}
$$

The possibility of changing the value of variables $a_{i a}^{b}$ or $r_{i a}^{b}$ is considered only if facility $i$ has remaining capacity greater than zero at the end of time period $b$ (otherwise, even if the facility was operational during time period $t$, it would not increase the total available capacity).

If a service is already open during time period $t$, there is the possibility of increasing its available capacity by (re) opening the facility before time period $t$. This can be achieved either by splitting variables $a_{i \tau}^{\xi}$ or $r_{i \tau}^{\xi}$, with $\tau \leq t$, that are considered equal to one in the present primal solution or by considering new variables $a_{i \tau}^{\xi}$ or $r_{i \tau}^{\xi}$ such that $i \notin I_{t^{\prime}}^{+}$, for $\tau \leq t^{\prime} \leq \xi<t$. The calculation of $p_{i}^{t}$ takes all these possibilities into account.

\section{Calculation of $p_{i}^{t}$ for $i \in I_{t}^{+}$}

1. $p_{i}^{t} \leftarrow \min \left\{\begin{array}{c}+\infty, \min _{(i, \tau, \xi) \in I_{A}^{+}}\left\{F A_{i \tau}^{\zeta}+F R_{i \zeta+1}^{\xi}-F A_{i \tau}^{\xi}\right\}, \\ \tau \leq t \\ \zeta \leq t\end{array}\right.$

$$
\left.\min _{\substack {(i, \tau, \xi) \in I_{R}^{+} \\
\begin{subarray}{c}{\tau \leq t \\
\zeta \leq t{ ( i , \tau , \xi ) \in I _ { R } ^ { + } \\
\begin{subarray} { c } { \tau \leq t \\
\zeta \leq t } }\end{subarray}}\left\{F R_{i \tau}^{\zeta}+F R_{i \zeta+1}^{\xi}-F R_{i \tau}^{\xi}\right\}\right\} .
$$

2. $t 1 \leftarrow 1$;

3. If $i \in I_{t 1}^{+}$go to 6 . Else continue.

4. $b=\max \left\{0, \max _{t^{\prime}<t 1}\left\{t^{\prime}: i \in I_{t^{\prime}}^{+}\right\}\right\} ; \quad c=\min \left\{t, \min _{t^{\prime}>t 1}\left\{t^{\prime}: i \in I_{t^{\prime}}^{+}\right\}\right\}$;

5. If $b=0$, there exists $(i, a, d) \in I_{A}^{+}$. Then $p_{i}^{t} \leftarrow \min \left\{p_{i}^{t}, \min _{b<\tau \leq \xi<c}\left\{F A_{i \tau}^{\xi}\right\}+F R_{i a}^{d}-F A_{i a}^{d}\right\}$. Else $p_{i}^{t} \leftarrow \min \left\{p_{i}^{t}\right.$, $\left.\min _{b<\tau \leq \xi<c}\left\{F R_{i \tau}^{\xi}\right\}\right\}$.

6. $t 1 \leftarrow t 1+1$; If $t 1=t$ then go to 7 . Else go to 3 .

7. $p_{i}^{t} \leftarrow \frac{p_{i}^{t}}{Q_{i}}\left\lceil\frac{\phi_{i}}{Q_{i}}\right\rceil$, where $\phi_{i}=\left\{\begin{array}{l}D-C, \text { if } C+Q_{i}<D \\ Q_{i} \text {, otherwise } \text {. Stop. }\end{array}\right.$ 


\section{Computational tests}

\subsection{Description of the computational experiments}

The primal-dual heuristic was tested with a set of randomly generated problems. The following values for $m, n$ and $T(n>m)$ were considered and, for each combination, 5 instances were generated (total of 270 problems):

\begin{tabular}{llllll}
\hline$n$ & 25 & 50 & 100 & 200 & 500 \\
$m$ & 5 & 10 & 20 & 50 & \\
$T$ & 5 & 10 & 20 & & \\
\hline
\end{tabular}

The data for the test problems were generated according to the following procedure:

1. Random generation of $(x, y)$ coordinates in the plane of the $m+n$ nodes of the network, according to a uniform distribution and considering a $500 \times 500$ square.

2. Random creation of arcs between the network nodes, with a probability of $75 \%$.

3. Creation of arcs (not created in step 2) between nodes such that the Euclidean distance from one another is less than 50 , with probability of $80 \%$.

4. For the first period, the costs associated with arcs are randomly generated according to a uniform distribution, in the interval [100,1100]. For $t>1$, the cost associated to the arc in period $t$ is equal to the cost in period $t-1$ plus a changing factor randomly generated corresponding to a variation between $-10 \%$ and $+10 \%$.

5. For each time period, calculation of the shortest path between each client and each facility, using the Floyd-Warshall algorithm.

6. For each facility $i$ and period $t$, consider tend $=t, \ldots, T$. For tend $=t$, the fixed costs for variables $a_{i t}^{\text {tend }}$ and $r_{i t}^{\text {tend }}$ are randomly generated according to a uniform distribution in the interval $[500,3,500]$. For tend $>t$, a factor between $0 \%$ and $10 \%$, that represents an increase in the fixed cost for tend-1, is randomly generated.

7. The maximum capacities and the clients demands in each time period are randomly generated as described in Saldanha da Gama (2002).

All experiments were carried out in a Pentium 4, 1.80 Ghz, running under Windows 2000 operating system, with a maximum of 2,000 MB of virtual memory and $260 \mathrm{Mb}$ of Ram. The heuristic was programmed using C-language and Microsoft Visual C++ compiler. The performance of the algorithm was compared with the performance of CPLEX, version 7.0.

CPLEX terminates without calculating the optimal solution whenever more than 2,100,000,000 nodes of the branch and bound tree are explored, or when the number of simplex iterations in a node exceeds 2,100,000,000, or when there is not enough memory to read the problem or when the execution time exceeds $200,000 \mathrm{~s}$.

After the execution of the primal-dual heuristic, a local search procedure was executed. Let:

$\mathrm{SOL}_{S}=$ set of solutions constituting the $k$-neighborhood of solution $S$;

$Z_{S}=$ primal objective function value considering solution $S$.

Definition 1 An admissible solution $S^{\prime}$ is said to be in the $k$-neighborhood of the admissible solution $S$ if and only if $S$ ' differs from $S$ by the insertion or removal of at most $k$ functioning continuous time periods to a service $i$. 
The local search procedure can be described as follows:

\section{Local search procedure}

1. $k \leftarrow 1 . S=$ current primal solution.

2. Calculate $S^{+} \in S O L_{S}$ such that $Z_{S^{+}}=\min _{S^{\prime} \in S O L_{S}}\left\{Z_{S^{\prime}}\right\}$

3. If $Z_{S^{+}}<Z_{S}$, then $S \leftarrow S^{+}$and go to 2 . Else continue.

4. $k \leftarrow k+1$. If $k>T$ then stop. Else go to 2 .

We also tested the performance of a lagrangean heuristic procedure that uses the subgradient optimization method applied to the lagrangean relaxation obtained by relaxing, in a lagrangean way, the capacity restrictions. An uncapacitated dynamic location problem is obtained that is optimized using the heuristics described in Dias et al. (2007). Then a primal procedure similar to the one described in this paper is used to find an admissible solution.

\subsection{Computational results}

Table 1 shows the quality of the primal solutions found by the heuristic. It shows the results obtained when the dual variables are initialised as described in Sect. 3 (columns 4-6). It also shows average results of the quality of the primal solutions obtained by the primal-dual heuristic after the execution of the local search procedure around the best solution found by the heuristic (columns 7-9). In the following columns the results presented were obtained when the dual variables are initialised by solving a linear programming problem, as described in Saldanha da Gama (2002). Table 2 shows the results obtained with the lagrangean heuristic described in the end of the previous section. The tables show the worst, the best and the average value of the deviations of the final primal solution found from the best known lower bound on the optimal value. This lower bound is equal to the optimum value for all problems CPLEX was able to solve. For all the others, this lower bound is given by the best dual solution found by the primal-dual heuristic. The values shown are calculated as $\left(Z-Z_{L B}\right) / Z_{L B}$, where $Z$ is the objective function value of the final primal solution found and $Z_{L B}$ is the value of the lower bound. In Tables 1 and 2 there are some values greater than $100 \%$. This happens for sets of problems that CPLEX was unable to solve and was not even capable of solving the linear relaxation. This means that the best lower bound known is the one given by the primal-dual heuristics. As can be seen in Table 3, that shows the quality of the dual solution calculated by the heuristics, the quality of this lower bound is very poor. The quality of the lower bound is calculated as $\left(Z^{*}-Z_{L B}\right) / Z^{*}$, where $Z^{*}$ represents the best upper bound known. That is why these values greater than $100 \%$ appear in Table 1 . Table 4 shows the computational times spent by the heuristics and by CPLEX. CPLEX is unable to solve one of the five problems with ( $T, n, m)$ equal to $(20,100,20)$ and is capable of solving only one of the five problems with $(T, n, m)$ equal to $(20,200,20)$. The symbol ' - ' is used in Table 3 for all cases where CPLEX was not capable of solving any of the five problems. 
Table 1 Quality of the primal solution in percentage (primal-dual heuristic)

\begin{tabular}{|c|c|c|c|c|c|c|c|c|c|c|c|c|c|c|}
\hline \multirow[t]{2}{*}{$T$} & \multirow[t]{2}{*}{$n$} & \multirow[t]{2}{*}{$m$} & \multicolumn{3}{|c|}{ Heuristic } & \multicolumn{3}{|c|}{$\begin{array}{l}\text { Heuristic + } \\
\text { local search }\end{array}$} & \multicolumn{3}{|c|}{$\begin{array}{l}\text { Heuristic with } \\
\text { Initialisation } \\
\text { using a LP }\end{array}$} & \multicolumn{3}{|c|}{$\begin{array}{l}\text { Heuristic with } \\
\text { Initialisation using } \\
\text { a LP + local search }\end{array}$} \\
\hline & & & Best & Average & Worst & Best & Average & Worst & Best & Average & Worst & Best & Average & Worst \\
\hline 5 & 25 & 5 & 0.22 & 1.17 & 3.47 & 0.00 & 0.12 & 0.62 & 0.24 & 2.21 & 4.95 & 0.00 & 1.26 & 4.88 \\
\hline 5 & 25 & 10 & 1.21 & 2.01 & 4.17 & 0.89 & 1.59 & 3.45 & 0.77 & 2.28 & 3.85 & 0.68 & 1.94 & 3.56 \\
\hline 5 & 25 & 20 & 1.46 & 2.72 & 3.89 & 1.40 & 2.12 & 3.26 & 1.06 & 2.53 & 3.87 & 0.41 & 2.02 & 3.51 \\
\hline 5 & 50 & 5 & 0.11 & 0.96 & 1.30 & 0.02 & 0.69 & 1.21 & 0.80 & 3.05 & 6.30 & 0.00 & 1.56 & 4.17 \\
\hline 5 & 50 & 10 & 1.31 & 2.93 & 4.68 & 1.30 & 2.43 & 3.40 & 1.46 & 2.22 & 3.62 & 0.74 & 1.51 & 1.98 \\
\hline 5 & 50 & 20 & 1.42 & 2.72 & 3.86 & 0.74 & 2.21 & 3.86 & 1.31 & 3.20 & 5.81 & 0.91 & 2.43 & 3.99 \\
\hline 5 & 100 & 5 & 0.42 & 1.75 & 4.65 & 0.10 & 1.38 & 3.86 & 0.00 & 2.38 & 4.57 & 0.00 & 2.10 & 4.46 \\
\hline 5 & 100 & 10 & 1.00 & 1.46 & 2.05 & 0.72 & 1.16 & 2.05 & 0.73 & 1.52 & 2.25 & 0.44 & 1.09 & 1.79 \\
\hline 5 & 100 & 20 & 1.40 & 2.44 & 3.49 & 1.40 & 1.99 & 2.89 & 0.87 & 2.03 & 3.24 & 0.63 & 1.59 & 3.24 \\
\hline 5 & 100 & 50 & 1.71 & 3.23 & 4.40 & 1.34 & 2.86 & 3.59 & 1.70 & 3.18 & 3.78 & 1.70 & 2.69 & 3.17 \\
\hline 5 & 200 & 5 & 0.03 & 1.45 & 2.82 & 0.00 & 0.77 & 2.02 & 0.03 & 1.38 & 2.82 & 0.01 & 1.11 & 2.12 \\
\hline 5 & 200 & 10 & 1.22 & 1.91 & 2.80 & 0.99 & 1.56 & 2.05 & 1.30 & 2.13 & 3.11 & 0.99 & 1.84 & 2.89 \\
\hline 5 & 200 & 20 & 1.76 & 2.61 & 3.88 & 1.41 & 2.21 & 3.88 & 0.97 & 2.16 & 3.69 & 0.68 & 1.71 & 2.56 \\
\hline 5 & 200 & 50 & 3.14 & 4.07 & 5.10 & 2.31 & 3.54 & 5.10 & 2.82 & 4.07 & 4.88 & 2.80 & 3.82 & 4.88 \\
\hline 5 & 500 & 5 & 0.00 & 0.66 & 1.38 & 0.00 & 0.55 & 1.18 & 0.00 & 1.00 & 2.84 & 0.00 & 0.72 & 1.76 \\
\hline 5 & 500 & 10 & 0.42 & 1.13 & 2.58 & 0.36 & 1.02 & 2.21 & 0.46 & 1.82 & 2.78 & 0.39 & 1.58 & 2.44 \\
\hline 5 & 500 & 20 & 0.78 & 2.32 & 3.44 & 0.70 & 1.79 & 3.13 & 1.11 & 1.94 & 2.93 & 0.99 & 1.55 & 2.21 \\
\hline 5 & 500 & 50 & 2.92 & 3.64 & 5.13 & 2.46 & 3.43 & 5.13 & 3.06 & 3.97 & 4.91 & 2.32 & 2.90 & 3.33 \\
\hline 10 & 25 & 5 & 0.76 & 1.32 & 2.35 & 0.09 & 0.78 & 117 & 0.83 & 2.07 & 5.70 & 0.54 & 1.75 & 5.52 \\
\hline 10 & 25 & 10 & 1.53 & 2.62 & 3.92 & 0.85 & 2.03 & 3.92 & 0.81 & 2.26 & 3.65 & 0.81 & 1.64 & 2.70 \\
\hline 10 & 25 & 20 & 2.20 & 3.52 & 4.69 & 1.46 & 2.47 & 3.73 & 2.98 & 3.97 & 5.07 & 1.33 & 2.49 & 3.88 \\
\hline 10 & 50 & 5 & 0.54 & 1.82 & 4.43 & 0.54 & 1.53 & 2.99 & 0.06 & 2.05 & 6.04 & 0.01 & 1.98 & 5.86 \\
\hline 10 & 50 & 10 & 1.79 & 2.02 & 2.57 & 1.08 & 1.72 & 2.57 & 0.76 & 2.12 & 3.99 & 0.52 & 1.39 & 1.92 \\
\hline 10 & 50 & 20 & 2.96 & 3.24 & 3.81 & 1.90 & 2.53 & 3.81 & 2.60 & 3.12 & 3.62 & 2.25 & 2.78 & 3.22 \\
\hline 10 & 100 & 5 & 0.16 & 0.70 & 1.26 & 0.01 & 0.42 & 0.99 & 0.26 & 1.27 & 2.57 & 0.14 & 0.68 & 2.00 \\
\hline 10 & 100 & 10 & 1.19 & 1.90 & 3.00 & 0.29 & 1.35 & 3.00 & 1.80 & 2.12 & 2.43 & 1.33 & 1.68 & 2.18 \\
\hline 10 & 100 & 20 & 1.92 & 2.45 & 2.91 & 1.15 & 1.95 & 2.91 & 1.75 & 2.39 & 5.00 & 1.21 & 1.99 & 2.40 \\
\hline 10 & 100 & 50 & 3.14 & 4.11 & 4.98 & 2.25 & 3.42 & 4.19 & 3.30 & 4.25 & 4.95 & 2.65 & 3.42 & 4.31 \\
\hline 10 & 200 & 5 & 0.23 & 1.52 & 2.26 & 0.01 & 1.11 & 2.19 & 0.23 & 1.31 & 2.14 & 0.23 & 1.13 & 1.51 \\
\hline 10 & 200 & 10 & 1.10 & 1.94 & 3.03 & 0.40 & 1.53 & 2.52 & 1.62 & 2.83 & 5.19 & 1.32 & 2.24 & 3.50 \\
\hline 10 & 200 & 20 & 2.05 & 2.94 & 3.53 & 1.22 & 1.73 & 2.38 & 1.94 & 3.07 & 3.62 & 1.94 & 2.57 & 3.14 \\
\hline 10 & 200 & 50 & 3.60 & 3.70 & 3.85 & 2.97 & 3.48 & 3.85 & 3.14 & 3.49 & 3.82 & 2.18 & 2.57 & 3.01 \\
\hline 10 & 500 & 5 & 0.14 & 0.75 & 1.36 & 0.01 & 0.58 & 1.33 & 0.41 & 0.88 & 1.19 & 0.41 & 0.76 & 0.99 \\
\hline 10 & 500 & 10 & 0.73 & 1.61 & 2.38 & 0.65 & 1.31 & 1.63 & 1.12 & 1.77 & 2.75 & 1.01 & 1.45 & 2.54 \\
\hline 10 & 500 & 20 & 1.80 & 2.26 & 2.83 & 1.43 & 1.66 & 2.05 & 1.68 & 2.38 & 3.18 & 1.17 & 2.07 & 2.62 \\
\hline 10 & 500 & 50 & 3.07 & 3.78 & 4.52 & 2.47 & 3.08 & 3.96 & 3.04 & 3.73 & 4.57 & 2.58 & 3.31 & 4.57 \\
\hline 20 & 25 & 5 & 1.45 & 3.09 & 4.51 & 1.36 & 2.13 & 3.29 & 1.32 & 3.64 & 5.24 & 1.21 & 2.37 & 2.77 \\
\hline 20 & 25 & 10 & 2.63 & 3.15 & 3.90 & 2.04 & 2.67 & 3.11 & 2.67 & 3.40 & 3.91 & 1.89 & 2.42 & 3.00 \\
\hline
\end{tabular}


Table 1 Continued

\begin{tabular}{|c|c|c|c|c|c|c|c|c|c|c|c|c|c|c|}
\hline \multirow[t]{2}{*}{$T$} & \multirow[t]{2}{*}{$n$} & \multirow[t]{2}{*}{$m$} & \multicolumn{3}{|c|}{ Heuristic } & \multicolumn{3}{|c|}{$\begin{array}{l}\text { Heuristic + } \\
\text { local search }\end{array}$} & \multicolumn{3}{|c|}{$\begin{array}{l}\text { Heuristic with } \\
\text { Initialisation } \\
\text { using a LP }\end{array}$} & \multicolumn{3}{|c|}{$\begin{array}{l}\text { Heuristic with } \\
\text { Initialisation using } \\
\text { a LP + local search }\end{array}$} \\
\hline & & & Best & Average & Worst & Best & Average & Worst & Best & Average & Worst & Best & Average & Worst \\
\hline 20 & 25 & 20 & 3.96 & 5.13 & 5.97 & 2.78 & 3.94 & 5.97 & 4.14 & 6.02 & 7.94 & 2.51 & 3.47 & 4.51 \\
\hline 20 & 50 & 5 & 1.47 & 2.55 & 4.34 & 0.50 & 1.77 & 3.40 & 0.67 & 2.32 & 4.64 & 0.28 & 1.49 & 3.06 \\
\hline 20 & 50 & 10 & 1.35 & 1.98 & 2.77 & 0.85 & 1.48 & 2.21 & 1.34 & 2.69 & 5.30 & 1.02 & 2.11 & 4.52 \\
\hline 20 & 50 & 20 & 4.17 & 4.77 & 5.48 & 3.99 & 4.62 & 5.48 & 3.92 & 4.48 & 5.31 & 2.08 & 3.07 & 3.92 \\
\hline 20 & 100 & 5 & 0.93 & 2.34 & 3.74 & 0.93 & 2.06 & 3.31 & 0.66 & 2.45 & 4.87 & 0.58 & 1.84 & 2.85 \\
\hline 20 & 100 & 10 & 2.63 & 3.11 & 3.65 & 2.06 & 2.63 & 3.20 & 2.66 & 3.20 & 4.50 & 1.62 & 2.56 & 3.07 \\
\hline 20 & 100 & 20 & 2.99 & 3.94 & 4.50 & 2.52 & 3.18 & 4.40 & 3.28 & 3.72 & 4.07 & 2.31 & 2.63 & 3.28 \\
\hline 20 & 100 & 50 & 109.91 & 128.20 & 137.60 & 108.56 & 127.02 & 136.43 & 120.99 & 130.55 & 156.65 & 110.39 & 131.50 & 139.25 \\
\hline 20 & 200 & 5 & 1.79 & 2.13 & 2.49 & 1.51 & 1.88 & 2.40 & 2.36 & 2.89 & 3.25 & 1.89 & 2.38 & 2.95 \\
\hline 20 & 200 & 10 & 1.10 & 1.91 & 2.70 & 0.96 & 1.69 & 2.21 & 2.61 & 2.91 & 3.10 & 2.25 & 2.58 & 2.76 \\
\hline 20 & 200 & 20 & 2.86 & 3.39 & 4.26 & 1.88 & 2.75 & 3.55 & 3.02 & 4.23 & 5.02 & 2.42 & 3.12 & 3.77 \\
\hline 20 & 200 & 50 & 115.41 & 132.04 & 151.36 & 113.99 & 130.83 & 150.07 & 120.25 & 136.96 & 165.30 & 119.25 & 128.59 & 162.20 \\
\hline 20 & 500 & 5 & 1.58 & 2.85 & 3.95 & 1.19 & 2.28 & 3.56 & 1.75 & 3.12 & 4.03 & 1.49 & 2.98 & 3.63 \\
\hline 20 & 500 & 10 & 2.43 & 3.04 & 4.50 & 1.42 & 2.02 & 2.28 & 2.23 & 4.15 & 5.11 & 2.23 & 3.51 & 4.25 \\
\hline 20 & 500 & 20 & 3.65 & 4.37 & 7.29 & 2.44 & 3.86 & 4.24 & 3.98 & 5.12 & 7.43 & 3.18 & 4.98 & 6.69 \\
\hline 20 & 500 & 50 & 118.87 & 143.93 & 165.74 & 116.27 & 141.29 & 162.08 & 123.60 & 152.60 & 163.97 & 122.55 & 151.07 & 161.87 \\
\hline
\end{tabular}

Table 2 Quality of the primal solution in percentage (lagrangean heuristics)

\begin{tabular}{|c|c|c|c|c|c|c|c|c|}
\hline \multirow[t]{2}{*}{$T$} & \multirow[t]{2}{*}{$n$} & \multirow[t]{2}{*}{$m$} & \multicolumn{3}{|c|}{ Lagrangean heuristic } & \multicolumn{3}{|c|}{$\begin{array}{l}\text { Lagrangean heuristic } \\
+ \text { local search }\end{array}$} \\
\hline & & & Best & Average & Worst & Best & Average & Wors \\
\hline 5 & 25 & 5 & 0.00 & 3.77 & 9.44 & 0.00 & 2.38 & 6.05 \\
\hline 5 & 25 & 10 & 0.91 & 5.59 & 7.58 & 0.86 & 4.35 & 6.98 \\
\hline 5 & 25 & 20 & 3.06 & 4.97 & 7.71 & 2.09 & 4.08 & 7.17 \\
\hline 5 & 50 & 5 & 0.00 & 1.58 & 4.68 & 0.00 & 1.13 & 2.56 \\
\hline 5 & 50 & 10 & 1.48 & 4.67 & 8.34 & 0.95 & 2.80 & 4.00 \\
\hline 5 & 50 & 20 & 3.19 & 5.49 & 9.73 & 1.52 & 4.51 & 8.58 \\
\hline 5 & 100 & 5 & 0.97 & 3.42 & 4.73 & 0.97 & 3.23 & 4.64 \\
\hline 5 & 100 & 10 & 2.62 & 6.05 & 13.04 & 1.88 & 4.51 & 10.47 \\
\hline 5 & 100 & 20 & 3.81 & 5.68 & 8.54 & 3.22 & 4.60 & 6.66 \\
\hline 5 & 100 & 50 & 3.66 & 5.47 & 8.04 & 3.03 & 4.65 & 7.58 \\
\hline 5 & 200 & 5 & 2.08 & 3.09 & 4.16 & 0.00 & 1.93 & 3.50 \\
\hline 5 & 200 & 10 & 3.36 & 4.64 & 6.69 & 2.33 & 3.82 & 6.23 \\
\hline 5 & 200 & 20 & 4.20 & 5.73 & 8.20 & 3.03 & 3.98 & 4.89 \\
\hline 5 & 200 & 50 & 5.19 & 6.28 & 7.40 & 4.03 & 5.11 & 6.15 \\
\hline 5 & 500 & 5 & 0.00 & 1.54 & 3.12 & 0.00 & 1.02 & 1.81 \\
\hline
\end{tabular}


Table 2 Continued

\begin{tabular}{|c|c|c|c|c|c|c|c|c|}
\hline \multirow[t]{2}{*}{$T$} & \multirow[t]{2}{*}{$n$} & \multirow[t]{2}{*}{$m$} & \multicolumn{3}{|c|}{ Lagrangean Heuristic } & \multicolumn{3}{|c|}{$\begin{array}{l}\text { Lagrangean heuristic } \\
+ \text { local search }\end{array}$} \\
\hline & & & Best & Average & Worst & Best & Average & Worst \\
\hline 5 & 500 & 10 & 0.57 & 3.85 & 6.53 & 0.44 & 2.66 & 4.96 \\
\hline 5 & 500 & 20 & 2.87 & 4.23 & 5.96 & 1.66 & 3.49 & 5.35 \\
\hline 5 & 500 & 50 & 4.66 & 6.03 & 7.04 & 3.89 & 4.56 & 5.39 \\
\hline 10 & 25 & 5 & 4.27 & 8.40 & 11.44 & 4.12 & 5.16 & 6.38 \\
\hline 10 & 25 & 10 & 4.73 & 7.89 & 10.78 & 3.13 & 4.95 & 6.67 \\
\hline 10 & 25 & 20 & 4.53 & 7.77 & 10.70 & 2.39 & 3.32 & 3.90 \\
\hline 10 & 50 & 5 & 4.63 & 7.81 & 15.96 & 3.23 & 6.35 & 15.61 \\
\hline 10 & 50 & 10 & 3.42 & 11.54 & 30.00 & 0.77 & 7.67 & 20.89 \\
\hline 10 & 50 & 20 & 5.92 & 7.84 & 10.15 & 3.28 & 5.40 & 9.46 \\
\hline 10 & 100 & 5 & 4.95 & 9.25 & 22.99 & 2.08 & 5.57 & 15.02 \\
\hline 10 & 100 & 10 & 3.45 & 6.51 & 13.56 & 2.60 & 4.63 & 9.72 \\
\hline 10 & 100 & 20 & 5.32 & 8.89 & 13.61 & 2.20 & 5.82 & 10.35 \\
\hline 10 & 100 & 50 & 5.31 & 6.61 & 8.23 & 3.74 & 4.52 & 5.17 \\
\hline 10 & 200 & 5 & 4.76 & 7.17 & 11.77 & 4.22 & 6.18 & 8.80 \\
\hline 10 & 200 & 10 & 3.24 & 6.12 & 8.45 & 2.45 & 4.39 & 6.32 \\
\hline 10 & 200 & 20 & 5.74 & 10.21 & 14.36 & 2.86 & 6.78 & 11.60 \\
\hline 10 & 200 & 50 & 5.02 & 7.15 & 7.84 & 4.02 & 5.05 & 5.37 \\
\hline 10 & 500 & 5 & 0.20 & 0.95 & 1.57 & 0.17 & 0.89 & 1.18 \\
\hline 10 & 500 & 10 & 1.89 & 2.35 & 3.86 & 1.32 & 2.15 & 3.28 \\
\hline 10 & 500 & 20 & 2.88 & 3.14 & 3.90 & 2.73 & 3.10 & 3.71 \\
\hline 10 & 500 & 50 & 5.62 & 6.04 & 7.32 & 4.21 & 5.12 & 5.42 \\
\hline 20 & 25 & 5 & 2.32 & 3.86 & 5.75 & 1.85 & 2.89 & 5.52 \\
\hline 20 & 25 & 10 & 3.91 & 4.52 & 4.96 & 3.17 & 3.24 & 3.47 \\
\hline 20 & 25 & 20 & 5.59 & 6.25 & 7.04 & 4.42 & 5.07 & 5.28 \\
\hline 20 & 50 & 5 & 1.24 & 3.57 & 6.29 & 1.11 & 2.48 & 5.09 \\
\hline 20 & 50 & 10 & 2.22 & 2.89 & 4.15 & 1.91 & 2.75 & 3.28 \\
\hline 20 & 50 & 20 & 6.50 & 6.75 & 7.24 & 5.65 & 6.21 & 6.52 \\
\hline 20 & 100 & 5 & 1.38 & 2.84 & 3.56 & 1.22 & 2.45 & 2.88 \\
\hline 20 & 100 & 10 & 4.50 & 4.81 & 5.92 & 3.60 & 4.21 & 4.44 \\
\hline 20 & 100 & 20 & 4.75 & 5.94 & 8.34 & 3.56 & 5.45 & 6.67 \\
\hline 20 & 100 & 50 & 135.26 & 142.50 & 158.58 & 128.96 & 139.54 & 149.66 \\
\hline 20 & 200 & 5 & 2.80 & 3.68 & 5.28 & 2.10 & 3.12 & 4.43 \\
\hline 20 & 200 & 10 & 1.72 & 2.98 & 3.64 & 1.63 & 2.46 & 2.87 \\
\hline 20 & 200 & 20 & 5.23 & 5.98 & 6.64 & 4.19 & 5.34 & 5.71 \\
\hline 20 & 200 & 50 & 125.21 & 142.35 & 166.54 & 122.15 & 134.77 & 163.25 \\
\hline 20 & 500 & 5 & 2.75 & 3.39 & 5.40 & 2.31 & 2.96 & 4.38 \\
\hline 20 & 500 & 10 & 3.84 & 4.21 & 4.44 & 3.34 & 3.68 & 3.86 \\
\hline 20 & 500 & 20 & 7.04 & 7.56 & 9.03 & 4.93 & 5.38 & 6.77 \\
\hline 20 & 500 & 50 & 125.80 & 151.80 & 164.85 & 123.54 & 148.99 & 162.80 \\
\hline
\end{tabular}


Table 3 Quality of the dual solution in percentage

\begin{tabular}{|c|c|c|c|c|c|c|c|c|c|c|c|}
\hline \multirow[t]{2}{*}{$T$} & \multirow[t]{2}{*}{$n$} & \multirow[t]{2}{*}{$m$} & \multicolumn{3}{|c|}{ Heuristic } & \multicolumn{3}{|c|}{$\begin{array}{l}\text { Heuristic with } \\
\text { LP initialisation }\end{array}$} & \multicolumn{3}{|c|}{ Lagrangean heuristic } \\
\hline & & & Best & Average & Worst & Best & Average & Worst & Best & Average & Worst \\
\hline 5 & 25 & 5 & 15.37 & 24.61 & 34.78 & 6.42 & 13.58 & 21.55 & 0.66 & 24.40 & 42.61 \\
\hline 5 & 25 & 10 & 16.85 & 25.93 & 30.60 & 7.66 & 11.17 & 14.31 & 1.42 & 31.82 & 43.69 \\
\hline 5 & 25 & 20 & 27.06 & 27.77 & 28.48 & 9.27 & 12.90 & 17.34 & 42.85 & 44.12 & 45.66 \\
\hline 5 & 50 & 5 & 13.81 & 25.93 & 42.56 & 7.66 & 13.60 & 27.78 & 2.54 & 20.83 & 47.52 \\
\hline 5 & 50 & 10 & 27.48 & 29.08 & 30.57 & 8.92 & 12.72 & 17.22 & 31.77 & 35.96 & 39.07 \\
\hline 5 & 50 & 20 & 22.80 & 27.60 & 32.87 & 7.68 & 12.32 & 19.68 & 32.86 & 37.10 & 41.30 \\
\hline 5 & 100 & 5 & 19.64 & 25.90 & 34.22 & 8.32 & 11.73 & 16.22 & 18.01 & 28.97 & 40.68 \\
\hline 5 & 100 & 10 & 21.12 & 29.14 & 39.52 & 7.31 & 13.23 & 22.07 & 29.15 & 36.49 & 46.93 \\
\hline 5 & 100 & 20 & 27.21 & 30.08 & 32.28 & 9.03 & 11.55 & 13.29 & 37.44 & 38.80 & 40.44 \\
\hline 5 & 100 & 50 & 28.81 & 30.91 & 32.39 & 13.56 & 14.32 & 15.57 & 40.22 & 43.43 & 48.50 \\
\hline 5 & 200 & 5 & 22.77 & 32.69 & 37.77 & 14.90 & 18.61 & 21.87 & 21.08 & 32.03 & 40.31 \\
\hline 5 & 200 & 10 & 28.37 & 34.25 & 42.09 & 8.85 & 16.13 & 28.39 & 27.84 & 37.55 & 48.64 \\
\hline 5 & 200 & 20 & 26.51 & 29.87 & 36.00 & 8.33 & 13.54 & 19.86 & 31.80 & 37.04 & 41.14 \\
\hline 5 & 200 & 50 & 31.12 & 35.29 & 37.98 & 15.36 & 18.13 & 21.23 & 42.39 & 45.33 & 46.95 \\
\hline 5 & 500 & 5 & 26.08 & 34.32 & 42.55 & 12.59 & 17.94 & 22.17 & 2.13 & 21.94 & 35.85 \\
\hline 5 & 500 & 10 & 30.13 & 36.73 & 46.93 & 15.34 & 18.28 & 24.36 & 28.29 & 32.75 & 36.39 \\
\hline 5 & 500 & 20 & 31.60 & 35.71 & 42.46 & 14.89 & 18.31 & 22.70 & 27.89 & 35.15 & 43.98 \\
\hline 5 & 500 & 50 & 33.76 & 36.10 & 39.87 & 15.11 & 18.08 & 21.07 & 42.01 & 44.13 & 47.48 \\
\hline 10 & 25 & 5 & 29.32 & 35.06 & 39.98 & 10.01 & 16.01 & 20.72 & 33.14 & 40.42 & 44.43 \\
\hline 10 & 25 & 10 & 31.63 & 36.93 & 46.23 & 12.34 & 16.44 & 22.67 & 38.27 & 43.31 & 52.12 \\
\hline 10 & 25 & 20 & 34.91 & 38.45 & 41.15 & 17.27 & 20.16 & 22.99 & 45.37 & 47.50 & 49.62 \\
\hline 10 & 50 & 5 & 24.70 & 34.44 & 49.43 & 10.09 & 16.10 & 25.01 & 29.34 & 38.33 & 52.85 \\
\hline 10 & 50 & 10 & 31.51 & 36.58 & 43.69 & 11.09 & 16.45 & 21.88 & 37.35 & 42.07 & 48.68 \\
\hline 10 & 50 & 20 & 37.27 & 42.21 & 44.35 & 13.34 & 20.36 & 24.41 & 44.01 & 49.28 & 51.52 \\
\hline 10 & 100 & 5 & 30.74 & 37.89 & 45.61 & 13.74 & 19.52 & 24.73 & 21.96 & 39.04 & 48.12 \\
\hline 10 & 100 & 10 & 35.83 & 38.59 & 41.84 & 13.74 & 18.92 & 22.22 & 25.89 & 39.41 & 47.86 \\
\hline 10 & 100 & 20 & 34.74 & 37.95 & 41.87 & 15.46 & 17.54 & 19.53 & 40.81 & 44.46 & 48.97 \\
\hline 10 & 100 & 50 & 41.19 & 42.06 & 43.98 & 20.76 & 21.59 & 23.10 & 49.17 & 50.44 & 52.51 \\
\hline 10 & 200 & 5 & 30.11 & 34.12 & 38.48 & 13.77 & 16.94 & 22.79 & 33.26 & 36.37 & 39.21 \\
\hline 10 & 200 & 10 & 35.48 & 39.41 & 44.36 & 17.69 & 34.27 & 91.55 & 35.72 & 40.81 & 44.93 \\
\hline 10 & 200 & 20 & 35.92 & 38.85 & 41.43 & 15.44 & 18.38 & 20.72 & 42.75 & 44.20 & 46.19 \\
\hline 10 & 200 & 50 & 38.66 & 40.58 & 42.15 & 16.31 & 19.56 & 22.78 & 48.87 & 53.78 & 64.89 \\
\hline 10 & 500 & 5 & 37.95 & 40.84 & 44.88 & 19.52 & 22.44 & 29.85 & 31.48 & 40.21 & 42.85 \\
\hline 10 & 500 & 10 & 35.29 & 42.90 & 51.35 & 16.46 & 22.82 & 32.07 & 45.96 & 46.89 & 47.98 \\
\hline 10 & 500 & 20 & 37.18 & 42.14 & 48.31 & 18.10 & 21.91 & 25.09 & 38.15 & 40.82 & 41.76 \\
\hline 10 & 500 & 50 & 42.55 & 45.88 & 48.33 & 21.60 & 24.94 & 27.56 & 37.28 & 42.17 & 44.69 \\
\hline 20 & 25 & 5 & 36.26 & 40.15 & 45.39 & 19.09 & 20.54 & 22.52 & 36.07 & 41.89 & 44.06 \\
\hline 20 & 25 & 10 & 41.03 & 43.95 & 46.20 & 20.46 & 22.50 & 24.32 & 27.60 & 35.25 & 41.21 \\
\hline 20 & 25 & 20 & 46.98 & 48.71 & 50.55 & 21.36 & 24.40 & 26.54 & 31.43 & 40.01 & 42.26 \\
\hline
\end{tabular}


Table 3 Continued

\begin{tabular}{|c|c|c|c|c|c|c|c|c|c|c|c|}
\hline \multirow[t]{2}{*}{$T$} & \multirow[t]{2}{*}{$n$} & \multirow[t]{2}{*}{$m$} & \multicolumn{3}{|c|}{ Heuristic } & \multicolumn{3}{|c|}{$\begin{array}{l}\text { Heuristic with } \\
\text { LP initialisation }\end{array}$} & \multicolumn{3}{|c|}{ Lagrangean heuristic } \\
\hline & & & Best & Average & Worst & Best & Average & Worst & Best & Average & Worst \\
\hline 20 & 50 & 5 & 33.73 & 43.10 & 50.21 & 15.99 & 21.93 & 28.18 & 35.25 & 41.89 & 44.88 \\
\hline 20 & 50 & 10 & 40.97 & 46.34 & 52.90 & 18.49 & 24.33 & 29.68 & 38.97 & 40.56 & 43.80 \\
\hline 20 & 50 & 20 & 45.08 & 48.14 & 50.65 & 22.16 & 23.70 & 26.19 & 28.65 & 38.10 & 42.83 \\
\hline 20 & 100 & 5 & 33.05 & 39.38 & 42.43 & 14.23 & 18.96 & 21.16 & 43.89 & 45.87 & 46.58 \\
\hline 20 & 100 & 10 & 42.60 & 48.22 & 53.90 & 20.62 & 23.81 & 29.20 & 40.69 & 42.54 & 44.58 \\
\hline 20 & 100 & 20 & 45.05 & 49.20 & 52.02 & 20.36 & 25.01 & 28.68 & 38.74 & 39.85 & 43.77 \\
\hline 20 & 100 & 50 & 52.05 & 55.85 & 57.70 & 32.54 & 36.45 & 42.26 & 26.52 & 32.58 & 46.82 \\
\hline 20 & 200 & 5 & 38.53 & 41.71 & 46.95 & 21.52 & 33.54 & 38.95 & 42.62 & 43.12 & 44.21 \\
\hline 20 & 200 & 10 & 39.87 & 48.77 & 57.88 & 37.59 & 39.78 & 48.48 & 40.90 & 42.03 & 43.51 \\
\hline 20 & 200 & 20 & 46.73 & 48.99 & 50.08 & 42.48 & 45.26 & 48.89 & 32.11 & 36.56 & 42.31 \\
\hline 20 & 200 & 50 & 47.80 & 49.22 & 51.80 & 43.54 & 47.95 & 49.25 & 37.02 & 39.60 & 46.25 \\
\hline 20 & 500 & 5 & 35.69 & 42.57 & 49.88 & 33.98 & 38.96 & 45.87 & 32.47 & 35.98 & 43.25 \\
\hline 20 & 500 & 10 & 37.55 & 42.69 & 51.49 & 34.58 & 39.48 & 48.54 & 41.50 & 43.13 & 44.62 \\
\hline 20 & 500 & 20 & 34.57 & 43.54 & 56.00 & 33.48 & 38.45 & 54.47 & 33.35 & 38.77 & 44.43 \\
\hline 20 & 500 & 50 & 42.69 & 44.52 & 58.10 & 43.02 & 46.15 & 53.87 & 37.46 & 38.99 & 39.83 \\
\hline
\end{tabular}

\subsection{Conclusions}

The analysis of the computational results allows the following conclusions:

1. The primal-dual heuristic developed is capable of calculating good quality solutions for the problem.

2. Initialising the dual variables by solving a linear programming problem decreases the quality of the best primal solution found.

3. The lagrangean heuristic calculates, on average, solutions that are worse than the primal-dual heuristics. This is a different result from the one obtained with the other capacitated problems studied by the authors.

4. The local search procedure can increase significantly the quality of the best primal solution found with a significant increase in the computational times.

5. The computational time spent by CPLEX is, on average, more than 10 times greater than the time spent by the heuristics. It is also interesting to note that most of the times CPLEX finishes without calculating the optimal solution to the problem. In average it calculates primal solutions that are $0.02 \%$ distant from the best lower bound known.

6. The heuristics presented are not capable of calculating good lower bounds. As can be seen in Table 2 , the lower bounds calculated are of very poor quality.

7. Only $0.03 \%$ of the linear relaxations of the problems generated have an optimal integer solution. 


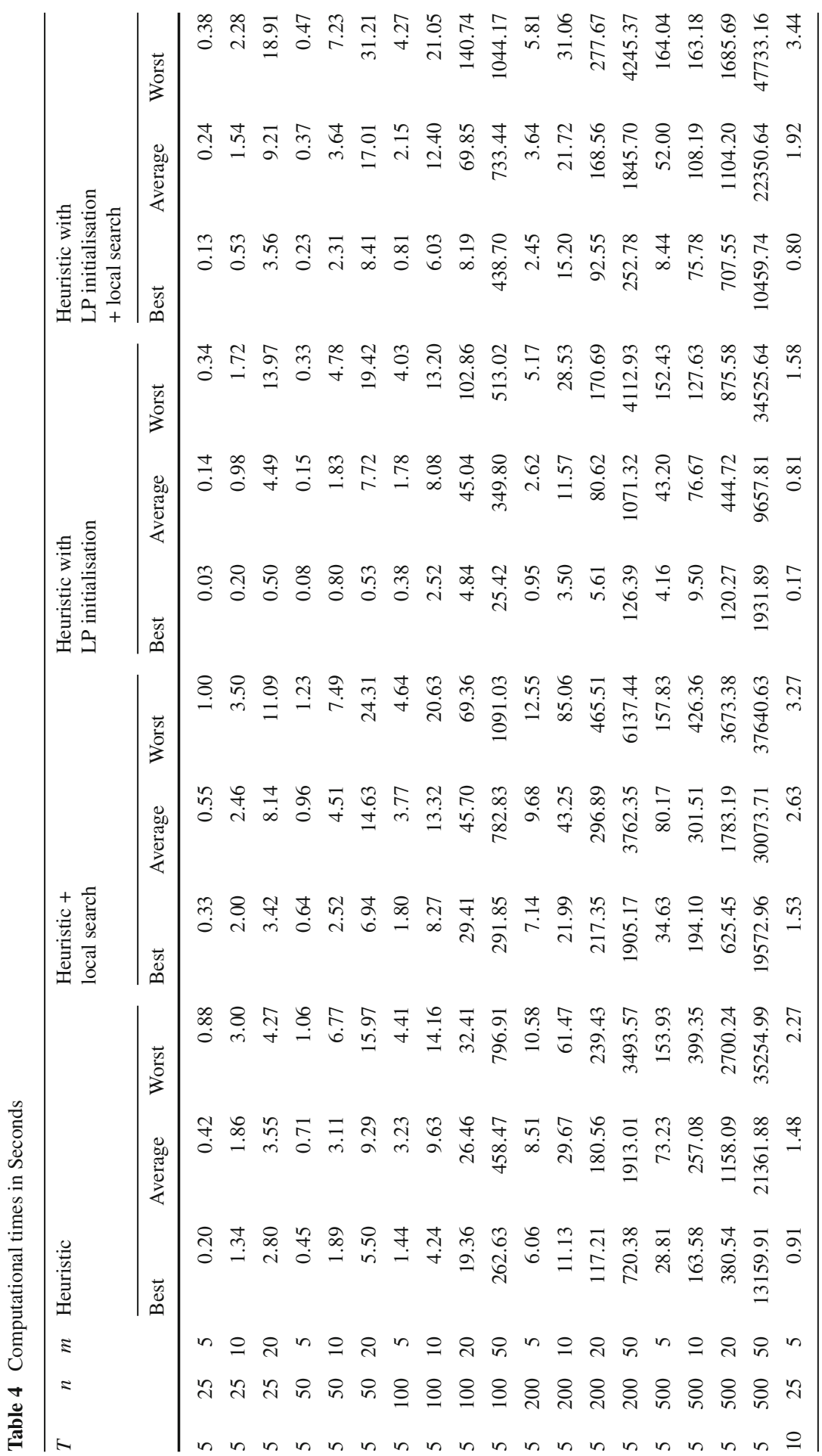




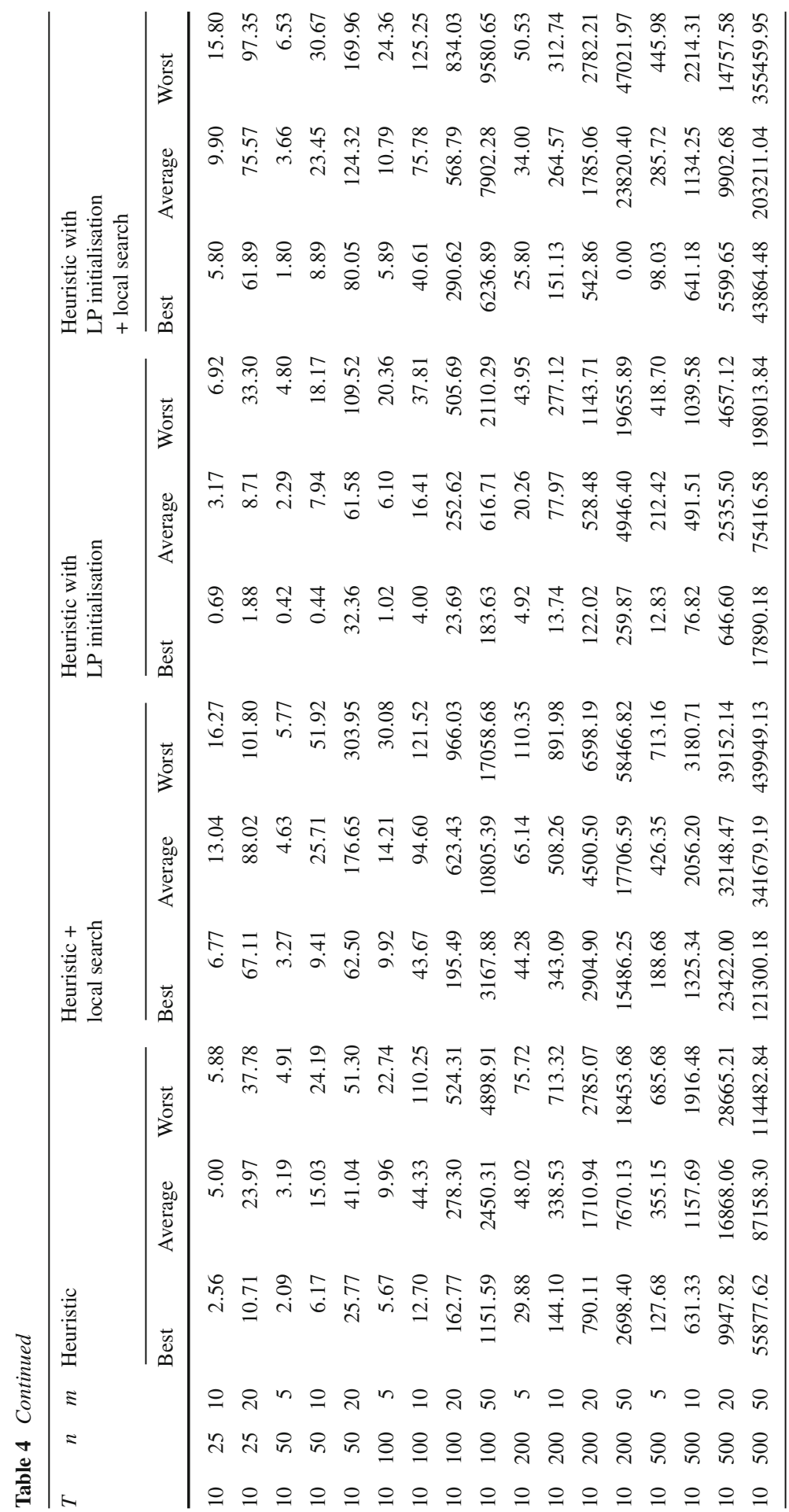




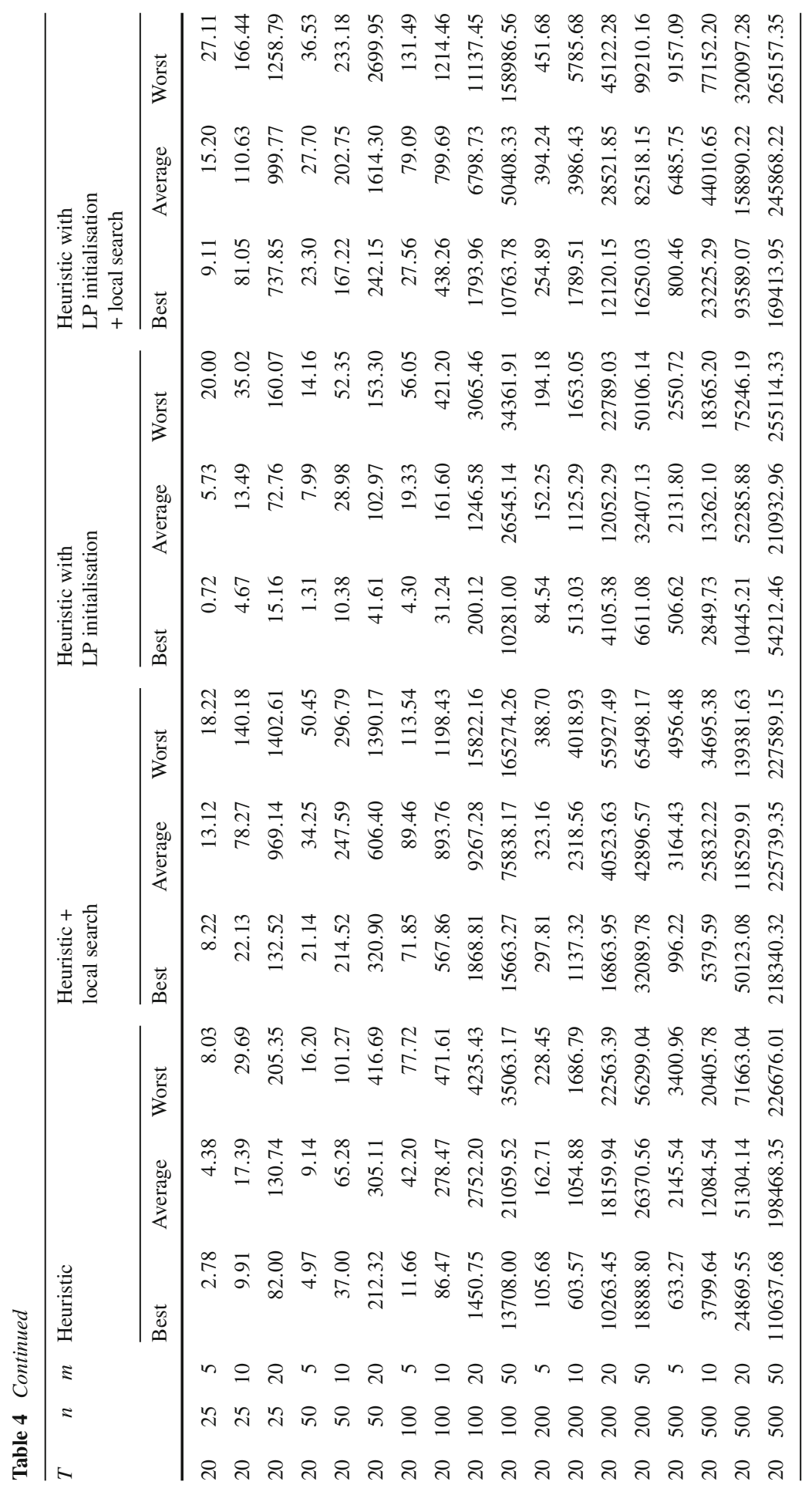




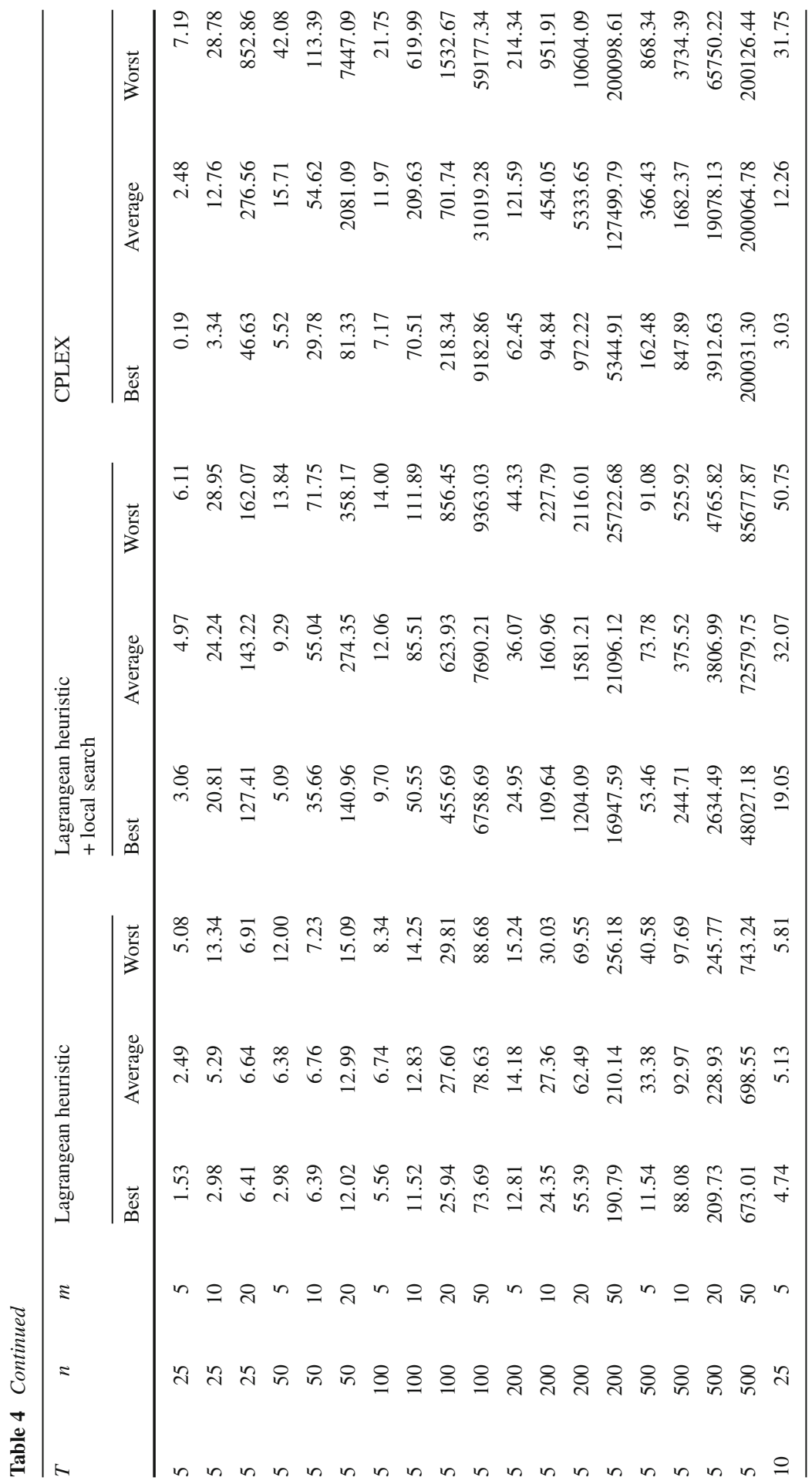




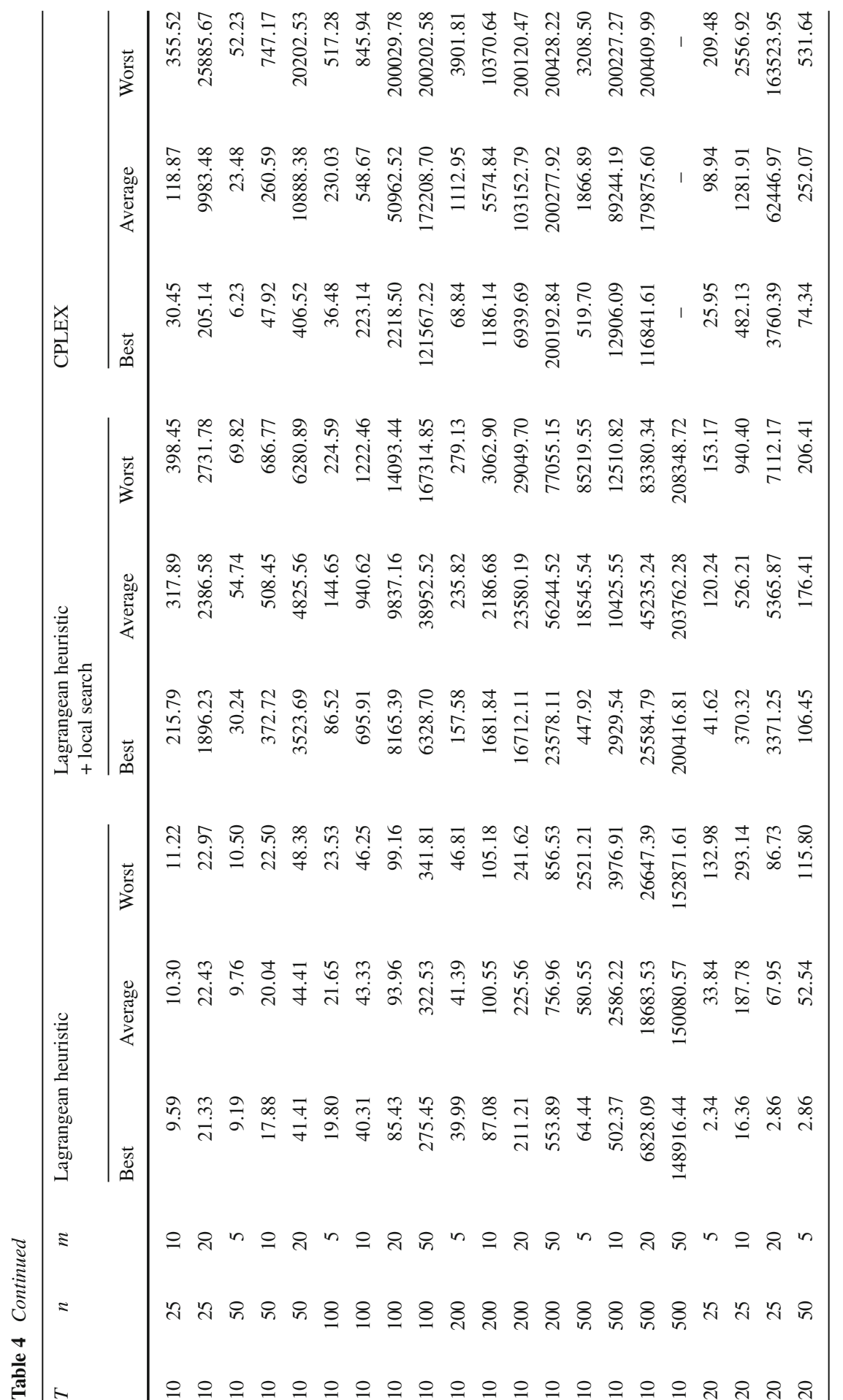




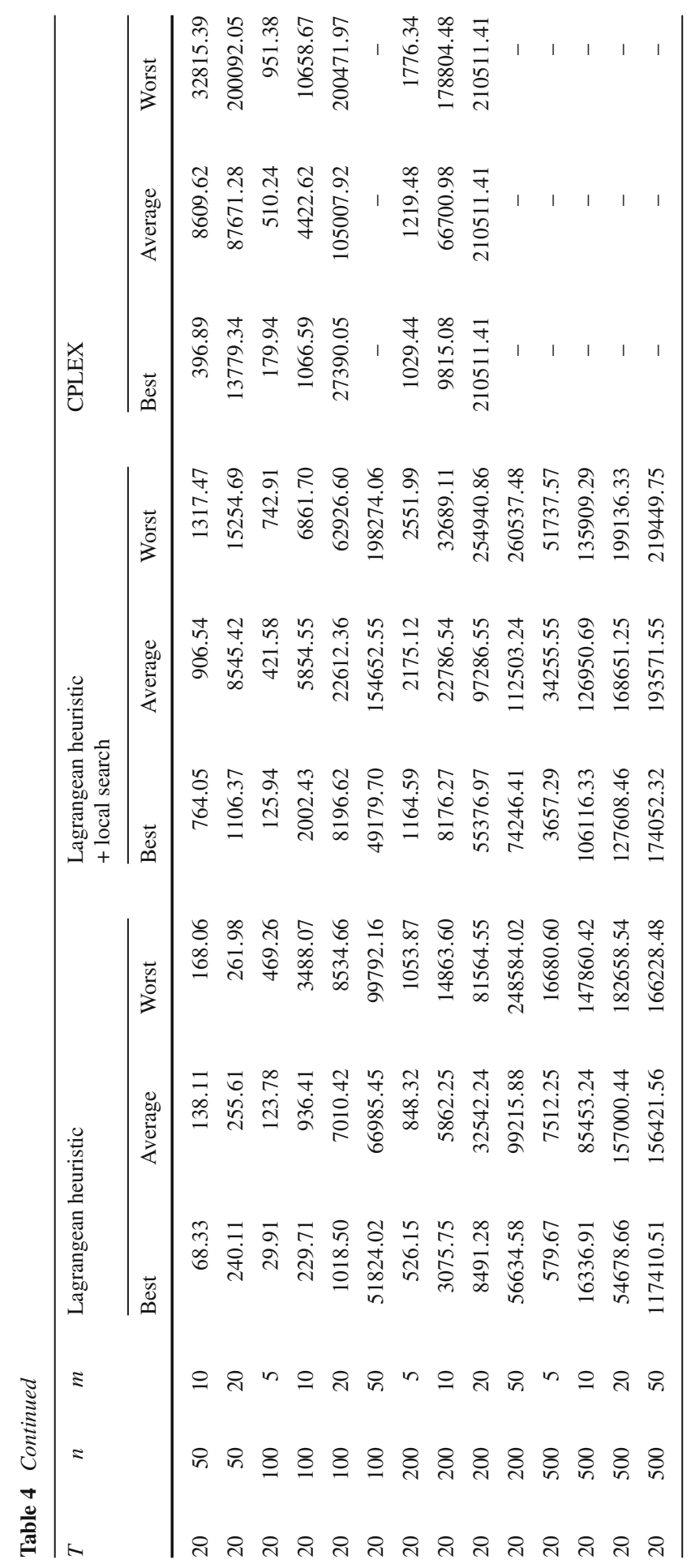




\section{Final comments and future work directions}

This work was motivated by the good results obtained with the computational tests performed with the primal-dual heuristic developed for the uncapacitated dynamic location problem (Dias et al. 2007). The computational tests already performed with the heuristic presented in this paper indicate that the primal solutions found by the heuristic are of good quality. The authors have also developed similar heuristics for multi-level capacitated and uncapacitated problems, and also for capacitated problems when the facilities can have different capacities.

\section{References}

Cornuejols G, Sridharan R, Thizy JM (1991) A comparison of heuristics and relaxations for the capacitated plant location problem. Eur J Oper Res 50:280-297

Dias J, Captivo ME, Clímaco J (2007) Efficient primal-dual heuristic for a dynamic location problem. Comput Oper Res 34:1800-1823

Dias J, Captivo, ME, Clímaco J (2006) Capacitated dynamic location problems with opening, closure and reopening of facilities. In: Salhi S, Drezner Z (eds) IMA J Math Manage Spec Issue Location Anal Appl Models 17:317-348

Erlenkotter D (1978) A dual-based procedure for uncapacitated facility location. Oper Res 26:992-1009

Guignard M, Spielberg K (1979) A direct dual method for the mixed plant location problem with some side constraints. Math Program 17:198-228

Saldanha da Gama F, Captivo ME (2002) A branch-and-bound procedure for the multi-period capacitated location problem. In: Working paper 8/2002, Centro de Investigação Operacional-Faculdade de Ciências da Universidade de Lisboa

Saldanha da Gama F (2002) Modelos e Algoritmos para o Problema de Localização Dinâmica, PhD Thesis, Faculdade de Ciências, Universidade de Lisboa (in Portuguese)

Sridharan R (1995) The capacitated plant location problem. Eur J Oper Res 87:203-213

Van Roy T, Erlenkotter D (1982) A dual-based procedure for dynamic facility location. Manage Sci 28(10):1091-1105 